\title{
STRICHARTZ ESTIMATES FOR THE KINETIC TRANSPORT EQUATION
}

\author{
EVGENI Y OVCHAROV
}

\begin{abstract}
In this paper we prove Strichartz estimates for the kinetic transport equation and make a detailed investigation on their range of validity. In one spatial dimension we find essentially all possible estimates, while in higher dimensions some endpoint and inhomogeneous estimates remain open. The remaining estimates are analogous to the remaining open inhomogeneous Strichartz estimates in other contexts. The Strichartz estimates that we derive extend the previous work by Castella and Perthame [5] (1996) and Keel and Tao [1] (1998) in the context of the kinetic transport equation, and the techniques of Foschi [7] to the current setting.
\end{abstract}

\section{INTRODUCTION}

The purpose of this paper is to study the range of validity of the Strichartz estimates for the kinetic transport (KT) equation

$$
\begin{aligned}
& \partial_{t} u(t, x, v)+v \cdot \nabla_{x} u(t, x, v)=F(t, x, v), \quad(t, x, v) \in(0, \infty) \times \mathbb{R}^{n} \times \mathbb{R}^{n}, \\
& u(0, x, v)=f(x, v) .
\end{aligned}
$$

The solution $u$ to (1), (2) has the form $u=U(t) f+W(t) F$, where

$$
U(t) f=f(x-t v, v), \quad W(t) F=\int_{-\infty}^{t} U(t-s) F(s) d s,
$$

and supp $F \subseteq(0, \infty)$. We want to study estimates of the form

$$
\|u\|_{L_{t}^{q} L_{x}^{r} L_{v}^{p}} \lesssim\|f\|_{L_{x, v}^{a}}+\|F\|_{L_{t}^{\tilde{q}^{\prime}} L_{x}^{\tilde{r}^{\prime}} L_{v}^{\tilde{p}^{\prime}}}
$$

where $L_{t}^{q} L_{x}^{r} L_{v}^{p}$ stands for the mixed Lebesgue space $L^{q}\left((0, \infty) ; L^{r}\left(\mathbb{R}^{n} ; L^{p}\left(\mathbb{R}^{n}\right)\right)\right)$, and $L_{x, v}^{a}$ stands for $L^{a}\left(\mathbb{R}^{2 n}\right)$. In the sequel we shall study separately the full range of Strichartz estimates for each operator $U(t)$ and $W(t)$. The Strichartz estimates for the KT equation of the form

$$
\|U(t) f\|_{L_{t}^{q} L_{x}^{r} L_{v}^{p}} \lesssim\|f\|_{L_{x, v}^{a}}
$$

are called homogeneous, while the estimates

$$
\|W(t) F\|_{L_{t}^{q} L_{x}^{r} L_{v}^{p}} \lesssim\|F\|_{L_{t}^{\tilde{q}^{\prime}} L_{x}^{\tilde{r}^{\prime}} L_{v}^{\tilde{p}^{\prime}}}
$$

are called inhomogeneous. As it is typically done, we shall prove estimates (3) and (41) under the slightly more general assumptions $t \in \mathbb{R}$ and $\operatorname{supp} F \subseteq(-\infty, \infty)$. For

Date: November 1, 2018.

2010 Mathematics Subject Classification. Primary: 35B45, Secondary: 35Q20.

Key words and phrases. Strichartz estimates, kinetic transport, dispersive.

Much of this work was done while at the University of Edinburgh. The author would like to thank Damiano Foschi for the several suggestions he made about this work. 
the sake of simplicity we shall again use the same notation $L_{t}^{q} L_{x}^{r} L_{v}^{p}$ for the space $L^{q}\left(\mathbb{R} ; L^{r}\left(\mathbb{R}^{n} ; L^{p}\left(\mathbb{R}^{n}\right)\right)\right)$.

Strichartz estimates for the KT equation appeared first in the note of Castella and Perthame [5] (1996) where a range of homogeneous estimates and some special inhomogeneous estimates are presented. In the seminal paper of Keel and Tao [1] (1998) the authors dedicate a small paragraph to the KT equation where they extend the homogeneous estimates proved in $[5$. However, the endpoint homogeneous estimate proves too difficult to be resolved by the methods presented in [11, which initiates an ongoing mathematical investigation. The first partial answer in that direction is given by Guo and Peng [8] (2007) who provide counterexamples in one spatial dimension confirming the (expected) failure of the endpoint estimate

$$
\|U(t) f\|_{L_{t}^{2} L_{x}^{\infty} L_{v}^{1}} \lesssim\|f\|_{L_{x, v}^{2}}
$$

there.

Presently, we extend the work of the previously mentioned authors by making a detailed analysis of the range of validity of the Strichartz estimates for the KT equation. The new estimates that we prove concern mostly the inhomogeneous operator $W(t)$ but we also prove new estimates for $U(t)$ of the more general form

$$
\|U(t) f\|_{L_{t}^{q} L_{x}^{r} L_{v}^{p}} \lesssim\|f\|_{L_{x}^{b} L_{v}^{c}} .
$$

In fact we prove that the latter estimates are equivalent to some special inhomogeneous estimates (4) which explains why the investigation of the inhomogeneous estimates is to us of primary interest.

As a motivation for studying Strichartz estimates for the KT equation we can point out the fact that they have been a very fruitful tool in the context of the wave and the Schrödinger equations in the analysis of the nonlinear Cauchy problem. Application of such type appeared in Bournaveas et al. 4] (2008) where the authors prove the existence of some weak solutions to a nonlinear kinetic system modeling chemotaxis.

The paper is organized as follows. In the next section we present the main results of the paper which we hope are given in a form convenient for referencing. In the section immediately after it we make some additional introductory remarks that

will be useful to those who wish to read on with our proofs. They follow in the sections after it.

\section{Strichartz estimates For the KT equation}

In order to formulate our results we first need several definitions.

Definition 2.1. We say that the exponent triplet $(q, r, p), 1 \leq p, q, r \leq \infty$, is KT-admissible if

$$
\begin{gathered}
\frac{1}{q}=\frac{n}{2}\left(\frac{1}{p}-\frac{1}{r}\right), \quad a \stackrel{\text { def }}{=} \operatorname{HM}(p, r), \\
1 \leq a \leq \infty, \quad p^{*}(a) \leq p \leq a, \quad a \leq r \leq r^{*}(a),
\end{gathered}
$$

except in the case $n=1,(q, r, p)=(a, \infty, a / 2)$.

In the above definition by $\operatorname{HM}(p, r)$ we have denoted the harmonic mean of $p$ and $r$, i.e. $a=\operatorname{HM}(p, r)$ whenever

$$
\frac{1}{a}=\frac{1}{2}\left(\frac{1}{r}+\frac{1}{p}\right) .
$$


For convenience we give explicitly the exact lower boundary $p^{*}$ to $p$ and the exact upper boundary $r^{*}$ to $r$ which are given in

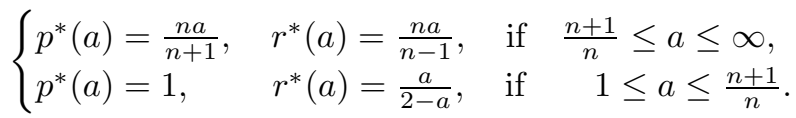

Note that the second line in (9) is placed to restrict the Lebesgue exponents $p$ and $r$ in the range $[1, \infty]$.

We have used above the convention that $1 / 0=\infty$, and thus, for example, for $n=1 r^{*}(a)=\infty$. Furthermore, throughout this text we shall always use the convention $1 / \infty=0$ and $1 / 0=\infty$ in the context of Lebesgue exponents. Triplets of the form $(q, r, p)=\left(a, r^{*}(a), p^{*}(a)\right)$, for $(n+1) / n \leq a<\infty$, will be called endpoint. The Hölder conjugate exponent will be denoted by ${ }^{\prime}$ e.g. $1 / r+1 / r^{\prime}=1$. Conditions (8), (9) are equivalent to $a \leq q$, and $p \leq r$, and $1 \leq a, p$, condition (7) is equivalent to

$$
\frac{1}{q}+\frac{n}{r}=\frac{n}{a}, \quad \operatorname{HM}(p, r)=a .
$$

Note that although the latter redaction of condition (7) resembles more closely the admissability conditions for the wave and the Schrödinger equations, the former version is more natural in the present context in view of the fact that in the inhomogeneous estimates the Lebesgue exponent $a$ does not appear explicitly.

To describe the range of the inhomogeneous estimates we shall need the next two definitions. Following Foschi [7, we give the following

Definition 2.2. We say that the exponent triplet $(q, r, p)$ is KT-acceptable if

$$
\frac{1}{q}<n\left(\frac{1}{p}-\frac{1}{r}\right), \quad 1 \leq q \leq \infty, \quad 1 \leq p<r \leq \infty,
$$

or if $q=\infty, 1 \leq p=r \leq \infty$.

Note that a KT-acceptable triplet is always KT-admissible. To further describe the range of validity of the inhomogeneous estimates we give the following

Definition 2.3. We say that the two KT-acceptable exponent triplets $(q, r, p)$ and $(\tilde{q}, \tilde{r}, \tilde{p})$ are jointly KT-acceptable if

$$
\begin{gathered}
\frac{1}{q}+\frac{1}{\tilde{q}}=n\left(1-\frac{1}{r}-\frac{1}{\tilde{r}}\right), \quad \frac{1}{q}+\frac{1}{\tilde{q}} \leq 1, \\
\operatorname{HM}(p, r)=\operatorname{HM}\left(\tilde{p}^{\prime}, \tilde{r}^{\prime}\right),
\end{gathered}
$$

and if the exponents satisfy further the additional restrictions

(i) for $r, \tilde{r} \neq \infty$

$$
\frac{n-1}{p^{\prime}}<\frac{n}{\tilde{r}}, \quad \frac{n-1}{\tilde{p}^{\prime}}<\frac{n}{r},
$$

(ii) if $r=\infty$ then the point $(1 / q, 1 / r, 1 / p, 1 / \tilde{q}, 1 / \tilde{r}, 1 / \tilde{p}) \in \Sigma_{1} \cup B$,

$$
\begin{aligned}
\Sigma_{1} & =\{(\mu, 0, \kappa, \nu, 1-\kappa, 1): 0<\mu, \nu<1,0<\mu+\nu<1, \kappa=(\mu+\nu) / n\}, \\
B & =(0,0,0,0,1,1),
\end{aligned}
$$

(iii) if $\tilde{r}=\infty$ then the point $(1 / q, 1 / r, 1 / p, 1 / \tilde{q}, 1 / \tilde{r}, 1 / \tilde{p}) \in \Sigma_{2} \cup C$,

$$
\begin{aligned}
\Sigma_{2} & =\{(\mu, 1-\kappa, 1, \nu, 0, \kappa): 0<\mu, \nu<1,0<\mu+\nu<1, \kappa=(\mu+\nu) / n\}, \\
C & =(0,1,1,0,0,0) .
\end{aligned}
$$


Conditions (14) and (15) are sharp which is demonstrated on counterexamples based on Besicovitch sets in Ovcharov [12].

Theorem 2.4. Let $u$ be the solution to the Cauchy problem for (11), (2). Then the estimate

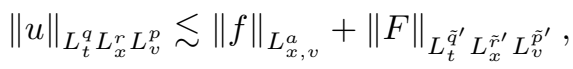

holds for all $f \in L_{x, v}^{a}$ and all $F \in L_{t}^{\tilde{q}^{\prime}} L_{x}^{\tilde{r}^{\prime}} L_{v}^{\tilde{p}^{\prime}}$ if and only if $(q, r, p)$ and $(\tilde{q}, \tilde{r}, \tilde{p})$ are two KT-admissible exponent triplets and $a=\operatorname{HM}(p, r)=\operatorname{HM}\left(\tilde{p}^{\prime}, \tilde{r}^{\prime}\right)$, apart from the case in higher dimensions $n>1$ of $(q, r, p)$ being an endpoint triplet for which the corresponding estimates remain unresolved.

Note that Theorem 2.4 allows the second triplet $(\tilde{q}, \tilde{r}, \tilde{p})$ to be endpoint and excludes only the estimates where the first triplet $(q, r, p)$ is endpoint. Below we employ the notation $L_{t}^{q} L_{x}^{r} L_{v}^{p}(V)$ for the Lebesgue space $L^{q}\left(\mathbb{R} ; L^{r}\left(\mathbb{R}^{n} ; L^{p}(V)\right)\right.$ ) (or $\left.L^{q}\left((0, \infty) ; L^{r}\left(\mathbb{R}^{n} ; L^{p}(V)\right)\right)\right)$ over a finite velocity domain $V \subset \mathbb{R}^{n}$.

Theorem 2.5 (Generalized inhomogeneous estimates). Suppose that ( $q, r, p)$ and $(\tilde{q}, \tilde{r}, \tilde{p})$ are two jointly KT-acceptable exponent triplets that further satisfy the following conditions

(i) $1<q, \tilde{q}<\infty, q>\tilde{q}^{\prime}$, then the estimate

$$
\|W(t) F\|_{L_{t}^{q} L_{x}^{r} L_{v}^{p}} \lesssim\|F\|_{L_{t}^{\tilde{q}^{\prime}} L_{x}^{\tilde{r}^{\prime}} L_{v}^{\tilde{p}^{\prime}}}
$$

holds for all $F \in L_{t}^{\tilde{q}^{\prime}} L_{x}^{\tilde{r}^{\prime}} L_{v}^{\tilde{p}^{\prime}}$.

(ii) $\tilde{q}=\infty, 1<q<\infty$, then the estimate

$$
\begin{aligned}
\|W(t) F\|_{L_{t}^{q, \infty} L_{x}^{r} L_{v}^{p}} & \lesssim\|F\|_{L_{t}^{1} L_{x}^{\tilde{r}^{\prime}} L_{v}^{\tilde{p}^{\prime}}} \\
\left(\|W(t) F\|_{L_{t}^{q} L_{x}^{r} L_{v}^{p}}\right. & \left.\lesssim\|F\|_{L_{t}^{\tilde{q}^{\prime}} L_{x}^{\tilde{r}^{\prime}} L_{v}^{\tilde{p}^{\prime}}}, \quad q \geq \tilde{p}^{\prime}\right)
\end{aligned}
$$

holds for all $F \in L_{t}^{1} L_{x}^{\tilde{r}^{\prime}} L_{v}^{\tilde{p}^{\prime}}$.

(iii) $q=\infty, 1<\tilde{q}<\infty$, then the estimate

$$
\begin{aligned}
&\|W(t) F\|_{L_{t}^{\infty} L_{x}^{r} L_{v}^{p}} \lesssim\|F\|_{L_{t}^{\tilde{q}^{\prime}, 1} L_{x}^{\tilde{r}^{\prime}} L_{v}^{\tilde{p}^{\prime}}}, \\
&\left(\|W(t) F\|_{L_{t}^{q} L_{x}^{r} L_{v}^{p}} \lesssim\|F\|_{L_{t}^{\tilde{q}^{\prime}} L_{x}^{\tilde{r}^{\prime}} L_{v}^{\tilde{p}^{\prime}}}, \quad \tilde{q}^{\prime} \leq p\right)
\end{aligned}
$$

holds for all $F \in L_{t}^{\tilde{q}^{\prime}, 1} L_{x}^{\tilde{r}^{\prime}} L_{v}^{\tilde{p}^{\prime}}\left(F \in L_{t}^{\tilde{q}^{\prime}} L_{x}^{\tilde{r}^{\prime}} L_{v}^{\tilde{p}^{\prime}}\right)$.

(iv) $1<q, \tilde{q}<\infty, q=\tilde{q}^{\prime}$. Under the assumption of a finite velocity space $V \subset \mathbb{R}^{n}$ we have that the estimate

$$
\begin{aligned}
\|W(t) F\|_{L_{t}^{q} L_{x}^{r, q} L_{v}^{P}(V)} & \lesssim V\|F\|_{L_{t}^{\tilde{q}^{\prime}} L_{x}^{\tilde{r}^{\prime}, \tilde{q}^{\prime}} L_{v}^{\tilde{P}^{\prime}}(V)} \\
\left(\|W(t) F\|_{L_{t}^{q} L_{x}^{r} L_{v}^{P}(V)}\right. & \left.\lesssim V\|F\|_{L_{t}^{\tilde{q}^{\prime}} L_{x}^{\tilde{r}^{\prime}} L_{v}^{\tilde{P}^{\prime}}(V)}, \quad q \leq r \text { and } \tilde{q} \leq \tilde{r}\right)
\end{aligned}
$$

holds for all $F \in L_{t}^{\tilde{q}^{\prime}} L_{x}^{\tilde{r}^{\prime}} L_{v}^{\tilde{p}^{\prime}}(V)$, whenever $P, \tilde{P}$ are such that $1 \leq P<p$ and $1 \leq \tilde{P}<\tilde{p}$.

Conversely, if estimate [17) holds for all $F \in L_{t}^{q^{\prime}} L_{x}^{r^{\prime}} L_{v}^{p^{\prime}}$, then $(q, r, p)$ and $(\tilde{q}, \tilde{r}, \tilde{p})$ must be two jointly KT-acceptable exponent triplets, apart from condition (13) whose necessity is not fully verified. 
Remark 2.6. As indicated, in the range $q \geq \tilde{p}^{\prime}$ estimate (18) can be strengthen by replacing the Lorentz norm $L^{q, \infty}$ by the Lebesgue norm $L^{q}$. Analogously, the Lorentz norm $L^{\tilde{q}^{\prime}, 1}$ in estimate (19) can be replaced by the Lebesgue norm $L^{\tilde{q}^{\prime}}$ in the range $\tilde{q}^{\prime} \leq p$. This is proved in Lemma 8.3. By the same token, in the range $q \leq r$ and $\tilde{q} \leq \tilde{r}$, estimate (20) implies its analogue in Lebesgue norms.

Remark 2.7. If we restrict ourselves to finite time intervals $[0, T]$, we have the continuous embeddings

$$
\begin{aligned}
& L^{q, r}([0, T]) \hookrightarrow L^{p}([0, T]), \quad q>p, 1 \leq q, p, r \leq \infty, \\
& L^{p}([0, T]) \hookrightarrow L^{q, r}([0, T]), \quad p>q, 1 \leq q, p, r \leq \infty,
\end{aligned}
$$

see [2, p. 217]. For example, let $(\infty, r, p)$ and $(\tilde{q}, \tilde{r}, \tilde{p})$ be such that estimate (19) holds and let $1 \leq \widetilde{Q}<\tilde{q}$. Then we have the local inhomogeneous estimate

$$
\|W(t) F\|_{L_{t}^{\infty}\left([0, T] ; L_{x}^{q} L_{v}^{r}\right)} \lesssim_{T}\|F\|_{L_{t}^{\tilde{Q}^{\prime}}\left([0, T] ; L_{x}^{\tilde{r}^{\prime}} L_{v}^{\tilde{p}^{\prime}}\right)}
$$

for any $0<T<\infty$ and any $F \in L_{t}^{\tilde{Q}^{\prime}}\left([0, T] ; L_{x}^{\tilde{r}^{\prime}} L_{v}^{\tilde{p}^{\prime}}\right)$.

Theorem 2.8 (The Equivalence Theorem).

A. The following three estimates

$$
\begin{aligned}
\|U(t) f\|_{L_{t}^{q} L_{x}^{r} L_{v}^{p}} & \lesssim\|f\|_{L_{x}^{b} L_{v}^{c}}, \quad & \forall f \in L_{x}^{b} L_{v}^{c}, \\
\|W(t) F\|_{L_{t}^{q} L_{x}^{r} L_{v}^{p}} & \lesssim\|F\|_{L_{t}^{1} L_{x}^{b} L_{v}^{c}}, \quad & \forall F \in L_{t}^{1} L_{x}^{b} L_{v}^{c}, \\
\|W(t) F\|_{L_{t}^{\infty} L_{x}^{b^{\prime}} L_{v}^{c^{\prime}}} & \lesssim\|F\|_{L_{t}^{q^{\prime}} L_{x}^{r^{\prime}} L_{v}^{p^{\prime}}}, & \forall F \in L_{t}^{q^{\prime}} L_{x}^{r^{\prime}} L_{v}^{p^{\prime}} .
\end{aligned}
$$

are equivalent whenever $1 \leq q, r, p, b, c \leq \infty$.

B. Whenever $b=c=2$ estimate (21) is equivalent to

$$
\|W(t) F\|_{L_{t}^{q} L_{x}^{r} L_{v}^{r^{\prime}}} \lesssim\|F\|_{L_{t}^{q^{\prime}} L_{x}^{r^{\prime}} L_{v}^{r}}, \quad \forall F \in L_{t}^{q^{\prime}} L_{x}^{r^{\prime}} L_{v}^{r} .
$$

As a direct consequence of Theorem 2.5 and the Equivalence Theorem we obtain

Corollary 2.9. We have the estimate

$$
\|U(t) f\|_{L_{t}^{q, \infty} L_{x}^{r} L_{v}^{p}} \lesssim\|f\|_{L_{x}^{b} L_{v}^{c}}
$$

for all $f \in L_{x}^{b} L_{v}^{c}$ whenever the exponent 5-vector $(q, r, p, b, c)$ satisfies the following conditions

$$
\begin{gathered}
\frac{1}{q}+\frac{n}{r}=\frac{n}{b}, \quad \operatorname{HM}(p, r)=\operatorname{HM}(b, c) \stackrel{\text { def }}{=} a, \\
p<b \leq a \leq c<r, \\
a \leq r<r^{*}(c),
\end{gathered}
$$

in the range $1<q<\infty, 1 \leq p, \tilde{p}, r, \tilde{r}<\infty$. Estimate (25) also holds whenever

$$
b=c=p=r, \quad q=\infty, \quad \text { (the transport estimate), }
$$

and whenever $b=p, c=r$, and $1 / q=n / p-n / r$ (an immediate consequence of the decay estimate (41)).

Theorem 2.10 (Generalized homogeneous estimates). We have the estimate

$$
\|U(t) f\|_{L_{t}^{q} L_{x}^{r} L_{v}^{p}} \lesssim\|f\|_{L_{x}^{b} L_{v}^{c}}
$$


for all $f \in L_{x}^{b} L_{v}^{c}$ whenever $q \geq c$ and the exponent 5-vector $(q, r, p, b, c)$ satisfies (26) - (28) in the range $0<q, r, p, b, c<\infty$ or condition (29) in the range $0<b \leq \infty$. Conversely, if estimate (30) holds for all $f \in L_{x}^{b} L_{v}^{c}$ then $(q, r, p, b, c)$ must satisfy conditions (26) and (27) in the range $1 \leq q<\infty, 1 \leq p, \tilde{p}, r, \tilde{r}<\infty$ or condition (29) in the range $0<b \leq \infty$.

The necessity of condition (28) remains open in parallel to that of condition (13) in the setting of the inhomogeneous estimates. Therefore, we cannot exclude the possibility of the existence of more estimates of the form (30) in dimensions $n>1$ in the case of $b \neq c$.

The Equivalence Theorem, part B, together with Theorem 2.5, part (iv), imply the following weaker substitute for the endpoint homogeneous Strichartz estimate over finite velocity spaces.

Corollary 2.11. Let $1 \leq P<p^{*}(a),(n+1) / n \leq a<\infty$, and let $V \subset \mathbb{R}^{n}$ be bounded. Then, the following estimate

$$
\|U(t) f\|_{L_{t}^{a} L_{x}^{r *(a)} L_{v}^{P}(V)} \lesssim_{V}\|f\|_{L_{x, v}^{a}},
$$

holds for all $f \in L_{x, v}^{a}$.

\section{General introductory remarks}

We owe the reader an explanation why our results do not follow from earlier works on Strichartz estimates. As it is well-known these estimates follow from two main ingredients, the decay and the energy estimates. Besides these, in the context of the KT equation, it is also necessary to assume a further structure condition that greatly increases the range of estimates we may prove. Consider the decay estimate

$$
\|U(t) f\|_{L_{x}^{\infty} L_{v}^{1}} \lesssim \frac{1}{|t|^{n}}\|f\|_{L_{x}^{1} L_{v}^{\infty}}, \quad \forall t \in \mathbb{R},
$$

for the KT equation. Note that the mixed Lebesgue norm in (32) creates difficulties in interpolation if one uses the real method. Moreover, the general results of Keel and Tao [11] and Taggart [16] are based on the real method and if applied to the present context produce Strichartz estimates in non-Lebesgue norms. Additional complication arises from the fact that the KT propagator $U(t)$ preserves a whole family of Lebesgue norms

$$
\|U(t) f\|_{L_{x, v}^{a}}=\|f\|_{L_{x, v}^{a}}, \quad \forall t \in \mathbb{R}, 0<a \leq \infty,
$$

and not just the $L^{2}$-norm (corresponding to the energy estimate in other contexts). To mark the different nature of estimate (33) we shall call it the transport estimate and any class $L_{x, v}^{a}$ for $1 \leq a \leq \infty$ we shall call a transport class. The transport estimate is a consequence of the special case $a=2$ in (33) and the following invariance of the homogeneous KT equation

$$
f \rightarrow f^{\alpha}, \quad U(t) f \rightarrow(U(t) f)^{\alpha}, \quad 0<\alpha<\infty .
$$

Furthermore, this invariance allows us to prove new homogeneous Strichartz estimates from already proven ones. In fact, the exponents in

$$
\|U(t) f\|_{L_{t}^{q} L_{x}^{r} L_{v}^{p}} \lesssim\|f\|_{L_{x}^{b} L_{v}^{c}}, \quad \forall f \in L_{x}^{b} L_{v}^{c},
$$

transform according to the rule

$$
(q, r, p, b, c) \rightarrow(\alpha q, \alpha r, \alpha p, \alpha b, \alpha c)
$$


and any two estimates whose exponents are related in such a way are equivalent. Note that there is no such convenient tool in the inhomogeneous setting.

To summarize, the Strichartz estimates that we shall prove in the sequel are consequences of the decay estimate (32), the transport estimate (33), and the structural assumption (34).

We would like next to highlight some special advances that we make in the present work. Most of all, we study the equivalence between different types of Strichartz estimates. One such result is the fact that in the context of the KT equation the Strichartz estimates for the operator $W(t)$ and that of $T T^{*}$,

$$
T T^{*} F=\int_{-\infty}^{\infty} U(t-s) F(s) d s
$$

are equivalent. This greatly simplifies the use of duality arguments and we do not any longer need the Christ-Kiselev lemma in order to deduce the inhomogeneous Strichartz estimates via the corresponding estimates for $T T^{*}$.

We also show that any homogeneous estimate has corresponding inhomogeneous estimates to which it is equivalent e.g.

$$
\begin{aligned}
\|U(t) f\|_{L_{t}^{q} L_{x}^{r} L_{v}^{p}} & \lesssim\|f\|_{L_{x}^{b} L_{v}^{c}}, \quad \forall f \in L_{x}^{b} L_{v}^{c}, \\
\|W(t) F\|_{L_{t}^{q} L_{x}^{r} L_{v}^{p}} & \lesssim\|F\|_{L_{t}^{1} L_{x}^{b} L_{v}^{c}}
\end{aligned}
$$

are equivalent, and more generally see Theorem 2.8 .

One possible application of this equivalence is in the study of the range of estimates (36). Since the proof of these estimates in the present context is an entirely new result, we shall give an example from the context of the Schrödinger equation. The estimate

$$
\left\|U_{s}(t) f\right\|_{L_{t}^{q} L_{x}^{r}} \lesssim\|f\|_{L_{x}^{p}}, \quad \forall f \in L_{x}^{p},
$$

where by $U_{s}(t)$ we have denoted the Schrödinger propagator, was investigated by $\mathrm{T}$. Kato [10] (1994) for $1<p \leq 2$. We obtain a larger range of such estimates in higher dimensions $n>2$, see our $\mathrm{PhD}$ Thesis [13. This improvement is essentially due to the fact that our approach benefits from the more recent advances introduced by Keel and Tao [1] and Foschi [7] in the inhomogeneous setting and, of course, the implication of (36) by (37).

The last special result to be considered here is concerned with the endpoint Strichartz estimates for the KT equation in higher dimensions as in Theorem 2.5] part (iv). We prove there a class of estimates with a loss of integrability that can be made arbitrary small compared to the original endpoint estimates. However, our estimates are given entirely in terms of Lebesgue norms which is useful in applications. Furthermore, we give a counterexample showing that there does not exist a family of perturbed local estimates in a "full neighborhood" around any given endpoint estimate. The existence of the latter is required by the methods of Keel and Tao [11 and Foschi [7, and hence why it has not been yet possible to resolve in the positive the endpoint estimates of the considered type.

The different cases in Theorem 2.5 can be visualized quite easily. Let us first remember that the Lebesgue space $L^{p}$ is best seen as a "function" of $1 / p$ rather than $p$ in the context of interpolation. Therefore, the range of validity of the estimate (17) corresponds to a region in $\mathbb{R}^{6}$ of points with coordinates $(1 / q, 1 / r, 1 / p, 1 / \tilde{q}, 1 / \tilde{r}, 1 / \tilde{p})$. The projection of that region over the $1 / q-1 / \tilde{q}$-plane is visualized in Figure 1 


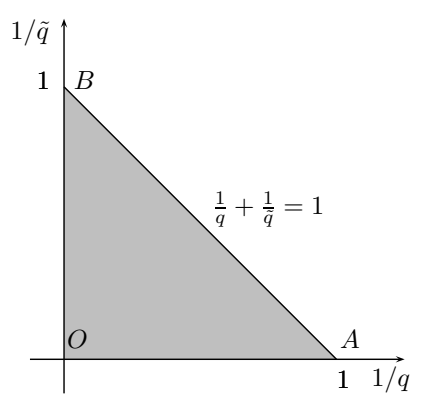

FigURE 1. Acceptable range of $(1 / q, 1 / \tilde{q})$.

The inner part of $\triangle O A B$ corresponds to the non-endpoint inhomogeneous estimates, while its three sides correspond to the endpoint inhomogeneous estimates. In the context of Theorem 2.5. the inner part of $\triangle O A B$ corresponds to part (i), the cathetus $O A$ - to part (ii), the cathetus $O B$ - to part (iii), and the hypotenuse $A B$ - to part (iv). The inhomogeneous estimates can be put into three groups in rising order of difficulty: the inner part of $\triangle O A B$, the two catheti $O A$ and $O B$, and the hypotenuse $A B$.

Note that in one spatial dimension condition (13) is void and thus there the complete range of validity of the Strichartz estimates for the KT equation is now known. In higher dimensions, however, the necessity (sharpness) of this condition is open. A similar question one encounters in other contexts, see e.g. Foschi [7] in the context of the Schrödinger equation.

Before we end this section we remark that the estimates that we prove remain valid for more general domains than those considered in the definition of equation (11). For example, the domain of $t$ may be any interval $I \subseteq \mathbb{R}$, and the domain of $v$ may be any measurable set $V \subseteq \mathbb{R}^{n}$. The claims follow from the fact that the transport and the dispersive estimate for the KT equation remain valid for these domains, as can one easily see by a simple modification of the proofs of Lemmas 4.1 and 4.2 .

The remainder of the paper is organized as follows. In the next section we give some auxiliary facts about the KT equation and in Section 5 we present the $T T^{*}$ method and some other duality arguments including the proof of the Equivalence Theorem 2.8. The proof of the Strichartz estimates for the Cauchy problem (Theorem 2.4) is given in Section 6. The local inhomogeneous Strichartz estimates are derived in Section 7 The generalized Strichartz estimates are proved in Section 8. In Section 9 we show sharpness of the estimates that we prove by means of counterexamples. We finish the paper with Section 10 where we list some still unanswered questions regarding the Strichartz estimates for the KT equation.

\section{Some PRoperties of the Kinetic transport EQUation}

Lemma 4.1 (The dispersive estimate [14]). The kinetic transport evolution group $U(t)$ obeys the estimate

$$
\|U(t) f\|_{L_{x}^{\infty} L_{v}^{1}} \leq \frac{1}{|t|^{n}}\|f\|_{L_{x}^{1} L_{v}^{\infty}},
$$


for all $f \in L_{x}^{1} L_{v}^{\infty}$.

Proof.

$$
\begin{array}{r}
\int_{\mathbb{R}^{n}}|U(t) f| d v=\int_{\mathbb{R}^{n}}|f(x-t v, v)| d v \leq \int_{\mathbb{R}^{n}} \sup _{y \in \mathbb{R}^{n}}|f(x-t v, y)| d v \\
\leq \frac{1}{|t|^{n}} \int_{\mathbb{R}^{n}} \sup _{y \in \mathbb{R}^{n}}|f(z, y)| d z=\frac{1}{|t|^{n}}\|f\|_{L_{x}^{1} L_{v}^{\infty}} .
\end{array}
$$

Lemma 4.2 (The transport estimate). The kinetic transport evolution group $U(t)$ obeys the estimate

$$
\|U(t) f\|_{L_{t}^{\infty} L_{x}^{a} L_{v}^{a}} \leq\|f\|_{L_{x, v}^{a}}, \quad 0<a \leq \infty
$$

for all $f \in L_{x, v}^{a}$.

Proof. Trivial.

Corollary 4.3 (The decay estimate). The kinetic transport evolution group $U(t)$ obeys the estimate

$$
\|U(t) f\|_{L_{x}^{r} L_{v}^{p}} \leq \frac{1}{|t|^{n\left(\frac{1}{p}-\frac{1}{r}\right)}}\|f\|_{L_{x}^{p} L_{v}^{r}}, \quad 1 \leq p \leq r \leq \infty,
$$

for all $f \in L_{x}^{p} L_{v}^{r}$.

Proof. Complex interpolation between the decay estimate (39) and the two transport estimates (40) with $a=1$ and $a=\infty$.

Lemma 4.4. The formal adjoint to $U(t)$ is the operator $U^{*}(t)=U(-t)$.

Proof. We denote by $\langle\cdot, \cdot\rangle$ the scalar product on $L^{2}\left(\mathbb{R}^{2 n}\right)$. Thus,

$$
\begin{aligned}
\langle U(t) f, g\rangle & =\int_{-\infty}^{\infty} f(x-t v, v) \overline{g(x, v)} d x d v \\
& =\int_{-\infty}^{\infty} f(y, v) \overline{g(y+t v, v)} d y d v=\langle f, U(-t) g\rangle,
\end{aligned}
$$

where we have made the substitution $y=x-t v$.

Lemma 4.5 (Scaling properties of $U(t)$ and $W(t)$ ). The evolution operators $U(t)$ and $W(t)$ enjoy the following scaling properties

$$
\begin{array}{r}
U(t) f_{\lambda}=f(x / \lambda-t v / \lambda, v)=\{U(\cdot) f\}(t / \lambda, x / \lambda, v), \\
\text { where } f_{\lambda}(x, v)=f(x / \lambda, v), \\
U(t) f_{\lambda}=f(x / \lambda-t v / \lambda, v / \lambda)=\{U(\cdot) f\}(t, x / \lambda, v / \lambda), \\
\text { where } f_{\lambda}(x, v)=f(x / \lambda, v / \lambda), \\
W(t) F_{\lambda}=\lambda \int_{-\infty}^{t / \lambda} F(s, x / \lambda-(t / \lambda-s) v, v) d s=\lambda\{W(\cdot) F\}(t / \lambda, x / \lambda, v), \\
\text { where } F_{\lambda}(t, x, v)=F(t / \lambda, x / \lambda, v), \\
W(t) F_{\lambda}=\int_{-\infty}^{t} F(s, x / \lambda-(t-s) v / \lambda, v / \lambda) d s=\{W(\cdot) F\}(t, x / \lambda, v / \lambda), \\
\text { where } F_{\lambda}(t, x, v)=F(t, x / \lambda, v / \lambda) .
\end{array}
$$


Proof. Direct inspection.

\section{Duality And The $T T^{*}$-PRINCIPle}

At the heart of the proof of Strichartz estimates lie duality arguments. In this section we introduce the main elements of all duality constructions in later proofs.

5.1. Basics. Let us consider the operator

$$
T: L_{x, v}^{2} \rightarrow L_{t}^{q} L_{x}^{r} L_{v}^{r^{\prime}}, \quad\{T f\}(t, x, v)=f(x-t v, v)=U(t) f,
$$

for some Lebesgue exponents $2 \leq q, r \leq \infty$. Its formal adjoint is the $L^{2}$-valued integral

$$
T^{*}: L_{t}^{q^{\prime}} L_{x}^{r^{\prime}} L_{v}^{r} \rightarrow L_{x, v}^{2}, \quad\left\{T^{*} F\right\}(x, v)=\int_{-\infty}^{\infty} F(s, x+s v, v) d s .
$$

The composition of the two has the form

$$
\begin{gathered}
T T^{*}: L_{t}^{q^{\prime}} L_{x}^{r^{\prime}} L_{v}^{r} \rightarrow L_{t}^{q} L_{x}^{r} L_{v}^{r^{\prime}} \\
\left\{T T^{*} F\right\}(t, x, v)=\int_{-\infty}^{\infty} F(s, x-(t-s) v, v) d s=\int_{-\infty}^{\infty} U(t-s) F(s) d s .
\end{gathered}
$$

In view of the $T T^{*}$-principle, see e.g. [15, p. 113], $T$ and $T T^{*}$ are equally bounded with $\|T\|^{2}=\left\|T T^{*}\right\|$. Thus, the following two estimates are equivalent

$$
\begin{array}{rlrl}
\|T f\|_{L_{t}^{q} L_{x}^{r} L_{v}^{r^{\prime}}} & \leq C\|f\|_{L_{x, v}^{2}}, \quad & \forall f \in L_{x, v}^{2}, \\
\left\|T T^{*} F\right\|_{L_{t}^{q} L_{x}^{r} L_{v}^{r^{\prime}}} & \leq C^{2}\|F\|_{L_{t}^{q^{\prime}} L_{x}^{r^{\prime}} L_{v}^{r}}, \quad \forall F \in L_{t}^{q^{\prime}} L_{x}^{r^{\prime}} L_{v}^{p^{\prime}},
\end{array}
$$

where $C=\|T\|$. We shall call (43) the symmetric $T T^{*}$-estimate. By $\langle\cdot, \cdot\rangle$ we denote the duality pairing on $\mathbb{R}^{2 n}$

$$
\langle f, g\rangle=\int_{R^{2 n}} f(x, v) g(x, v) d x d v
$$

for the (mixed) Lebesgue spaces. Thus, in bilinear formulation (43) reads

$$
\left|\int_{-\infty}^{\infty}\left\langle\left\{T T^{*} F\right\}(t), G(t)\right\rangle d t\right| \leq C^{2}\|F\|_{L_{t}^{q^{\prime}} L_{x}^{r^{\prime}} L_{v}^{r}}\|G\|_{L_{t}^{q^{\prime}} L_{x}^{r^{\prime}} L_{v}^{r}} .
$$

In view of Lemma 4.4, this is equivalent to

$$
\left|\int_{-\infty}^{\infty} \int_{-\infty}^{\infty}\left\langle U(s)^{*} F, U(t)^{*} G\right\rangle d s d t\right| \leq C^{2}\|F\|_{L_{t}^{q^{\prime}} L_{x}^{r^{\prime}} L_{v}^{r}}\|G\|_{L_{t}^{q^{\prime}} L_{x}^{r^{\prime}} L_{v}^{r}}
$$

$\forall F, \forall G \in L_{t}^{q^{\prime}} L_{x}^{r^{\prime}} L_{v}^{r}$. In 11] Keel and Tao noted that by symmetry, i.e. by changing the roles of $F$ and $G$, the latter estimate is always implied by the estimate

$$
|B(F, G)| \leq C^{2}\|F\|_{L_{t}^{q^{\prime}} L_{x}^{r^{\prime}} L_{v}^{r}}\|G\|_{L_{t}^{q^{\prime}} L_{x}^{r^{\prime}} L_{v}^{r}}, \quad \forall F, \forall G \in L_{t}^{q^{\prime}} L_{x}^{r^{\prime}} L_{v}^{r},
$$

for the bilinear form

$$
B(F, G)=\iint_{s<t}\left\langle U(s)^{*} F, U(t)^{*} G\right\rangle d s d t .
$$

Furthermore, we shall prove in the special context of the KT equation that these two estimates are in fact equivalent, see Lemma 5.1. 
We now consider the inhomogeneous estimates. Suppose that $(q, r, p)$ and $(\tilde{q}, \tilde{r}, \tilde{p})$ are two exponent triplets such that

$$
\begin{array}{ll}
\|T f\|_{L_{t}^{q} L_{x}^{r} L_{v}^{p}} \leq C\|f\|_{L_{x, v}^{a}}, & \forall f \in L_{x, v}^{a}, \\
\|T f\|_{L_{t}^{\tilde{q}} L_{x}^{\tilde{r}} L_{v}^{\tilde{v}}} \leq C\|f\|_{L_{x, v}^{a^{\prime}}}, & \forall f \in L_{x, v}^{a^{\prime}},
\end{array}
$$

for some $1 \leq a \leq \infty$. The composition

$$
L_{t}^{\tilde{q}^{\prime}} L_{x}^{\tilde{r}^{\prime}} L_{v}^{\tilde{p}^{\prime}} \stackrel{T^{*}}{\rightarrow} L_{x, v}^{a} \stackrel{T}{\rightarrow} L_{t}^{q} L_{x}^{r} L_{v}^{p}
$$

is bounded and thus

$$
\left\|T T^{*} F\right\|_{L_{t}^{q} L_{x}^{r} L_{v}^{p}} \leq C^{2}\|F\|_{L_{t}^{\tilde{q}^{\prime}} L_{x}^{\tilde{r}^{\prime}} L_{v}^{\tilde{p}^{\prime}}}, \quad \forall F \in L_{t}^{\tilde{q}^{\prime}} L_{x}^{\tilde{r}^{\prime}} L_{v}^{\tilde{p}^{\prime}} .
$$

This is the general non-symmetric $T T^{*}$-estimate for the KT equation. It does not any longer imply boundedness for the operator $T$, but as we shall see next, it implies boundedness for the operator $W(t) F$.

Lemma 5.1. In the context of the KT equation the non-symmetric TT*-estimate and the inhomogeneous Strichartz estimates are equivalent, i.e (45) is equivalent to

$$
\|W(t) F\|_{L_{t}^{q} L_{x}^{r} L_{v}^{p}} \leq C^{2}\|F\|_{L_{t}^{\tilde{q}^{\prime}} L_{x}^{\tilde{r}^{\prime}} L_{v}^{\tilde{p}^{\prime}}}, \quad \forall F \in L_{t}^{\tilde{q}^{\prime}} L_{x}^{\tilde{r}^{\prime}} L_{v}^{\tilde{p}^{\prime}} .
$$

Proof. (i) In one direction the claim follows immediately from

$$
|W(t) F| \leq T T^{*}|F| \text {. }
$$

(ii) In the other direction we have the following argument. It is easy to see that by duality (46) is equivalent to

$$
\begin{aligned}
|B(F, G)| \lesssim\|F\|_{L_{t}^{\tilde{q}^{\prime}} L_{x}^{\tilde{r}^{\prime}} L_{v}^{\tilde{p}^{\prime}}}\|G\|_{L_{t}^{q^{\prime}} L_{x}^{r^{\prime}} L_{v}^{p^{\prime}}} \\
\forall F \in L_{t}^{\tilde{q}^{\prime}} L_{x}^{\tilde{r}^{\prime}} L_{v}^{\tilde{p}^{\prime}}, \forall G \in L_{t}^{q^{\prime}} L_{x}^{r^{\prime}} L_{v}^{p^{\prime}} .
\end{aligned}
$$

By making the substitution $\sigma=-s, \tau=-t$ in the definition of $B(F, G)$ we get

$$
B(F, G)=\iint_{\tau<\sigma}\left\langle U(-\sigma)^{*} F, U(-\tau)^{*} G\right\rangle d \tau d \sigma .
$$

The integral in the line above can be written as

$$
(-1)^{n} \iint_{\tau<\sigma}\left\langle U(\sigma)^{*} F^{\prime}, U(\tau)^{*} G^{\prime}\right\rangle d \tau d \sigma \stackrel{\text { def }}{=}(-1)^{n} B^{\prime}\left(F^{\prime}, G^{\prime}\right),
$$

by making the substitution $x \rightarrow-x$ and setting $F^{\prime}(t, x, v)=F(-t,-x, v), G^{\prime}(t, x, v)$ $=G(-t,-x, v)$. Thus the boundedness of the bilinear form $B(\cdot, \cdot)$ implies the boundedness of the bilinear form $B^{\prime}(\cdot, \cdot)$ on the same spaces. The claim follows from the fact that the boundedness of $T T^{*}$ is equivalent to that of the bilinear form $B+B^{\prime}$.

For convenience we summarize the bilinear formulation of the duality arguments of this paragraph in

\section{Lemma 5.2.}

(i) The boundedness of the operator $T: L_{x, v}^{2} \rightarrow L_{t}^{q} L_{x}^{r} L_{v}^{r^{\prime}}$ of the form $T f=$ $U(t) f$ is equivalent to the boundedness of the bilinear mapping $B: L_{t}^{q^{\prime}} L_{x}^{r^{\prime}} L_{v}^{r}$ $\times L_{t}^{q^{\prime}} L_{x}^{r^{\prime}} L_{v}^{r} \rightarrow \mathbb{C}$. 
(ii) The boundedness of the operators $W(t): L_{t}^{\tilde{q}^{\prime}} L_{x}^{\tilde{r}^{\prime}} L_{v}^{\tilde{p}^{\prime}} \rightarrow L_{t}^{q} L_{x}^{r} L_{v}^{p}$ and $T T^{*}$ : $L_{t}^{\tilde{q}^{\prime}} L_{x}^{\tilde{r}^{\prime}} L_{v}^{\tilde{p}^{\prime}} \rightarrow L_{t}^{q} L_{x}^{r} L_{v}^{p}$ is equivalent to that of the bilinear mapping $B$ : $L_{t}^{\tilde{q}^{\prime}} L_{x}^{\tilde{r}^{\prime}} L_{v}^{\tilde{p}^{\prime}} \times L_{t}^{q^{\prime}} L_{x}^{r^{\prime}} L_{v}^{p^{\prime}} \rightarrow \mathbb{C}$.

\subsection{Equivalence of Strichartz estimates.}

Lemma 5.3 (The Duality lemma). The following two estimates for $W(t)$ are equivalent

$$
\begin{array}{ll}
\|W(t) F\|_{L_{t}^{q} L_{x}^{r} L_{v}^{p}} \lesssim\|F\|_{L_{t}^{\tilde{q}^{\prime}} L_{x}^{\tilde{r}^{\prime}} L_{v}^{\tilde{p}^{\prime}}}, \quad \forall F \in L_{t}^{\tilde{q}^{\prime}} L_{x}^{\tilde{r}^{\prime}} L_{v}^{\tilde{p}^{\prime}}, \\
\|W(t) F\|_{L_{t}^{\tilde{q}} L_{x}^{\tilde{r}} L_{v}^{\tilde{p}}} \lesssim\|F\|_{L_{t}^{q^{\prime}} L_{x}^{r^{\prime}} L_{v}^{p^{\prime}}}, \quad \forall F \in L_{t}^{q^{\prime}} L_{x}^{r^{\prime}} L_{v}^{p^{\prime}},
\end{array}
$$

for $1 \leq p, q \leq \infty$.

Proof. It follows immediately from the fact the boundedness of $W(t)$ is equivalent to that of the symmetric operator $T T^{*}$ on the considered spaces.

Theorem 5.4 (The Equivalence Theorem).

(i) The following three estimates

$$
\begin{array}{rlrl}
\|U(t) f\|_{L_{t}^{q} L_{x}^{r} L_{v}^{p}} & \lesssim\|f\|_{L_{x}^{b} L_{v}^{c}}, & & \forall f \in L_{x}^{b} L_{v}^{c}, \\
\|W(t) F\|_{L_{t}^{q} L_{x}^{r} L_{v}^{p}} & \lesssim\|F\|_{L_{t}^{1} L_{x}^{b} L_{v}^{c}}, & \forall F \in L_{t}^{1} L_{x}^{b} L_{v}^{c}, \\
\|W(t) F\|_{L_{t}^{\infty} L_{x}^{b^{\prime}} L_{v}^{c^{\prime}}} & \lesssim\|F\|_{L_{t}^{q^{\prime}} L_{x}^{r^{\prime}} L_{v}^{p^{\prime}}}, & \forall F \in L_{t}^{q^{\prime}} L_{x}^{r^{\prime}} L_{v}^{p^{\prime}} .
\end{array}
$$

are equivalent whenever $1 \leq q, r, p, b, c \leq \infty$.

(ii) Whenever $b=c=2$ estimate (48) is equivalent to

$$
\|W(t) F\|_{L_{t}^{q} L_{x}^{r} L_{v}^{r^{\prime}}} \lesssim\|F\|_{L_{t}^{q^{\prime}} L_{x}^{r^{\prime}} L_{v}^{r}}, \quad \forall F \in L_{t}^{q^{\prime}} L_{x}^{r^{\prime}} L_{v}^{r} .
$$

Proof. (i) The homogeneous estimate (48) trivially implies the first inhomogeneous estimate (49). In view of the Duality lemma 5.3, the two inhomogeneous estimates (49) and (50) are equivalent. All it remains to show is that (49) implies (48).

Let us first give a short formal proof. We choose $F(t)=\delta(t) f$ where $\delta(t)$ is the delta function on $\mathbb{R}$ and $f \in L_{x}^{b} L_{v}^{c}$. Consequently, $W(t)[\delta(\cdot) f]=U(t) f$ and thus

$$
\|U(t) f\|_{L_{t}^{q} L_{x}^{r} L_{v}^{p}} \lesssim\|\delta(t) f\|_{L_{t}^{1} L_{x}^{b} L_{v}^{c}}=\|f\|_{L_{x}^{b} L_{v}^{c}} .
$$

To make that rigorous instead of $\delta(t)$ we consider a smooth approximation of the identity $\delta_{\epsilon}(t)$, for $\epsilon>0$. So we are given the estimate

$$
|B(F, G)| \lesssim\|F\|_{L_{t}^{1} L_{x}^{b} L_{v}^{c}}\|G\|_{L_{t}^{q^{\prime}} L_{x}^{r^{\prime}} L_{v}^{p^{\prime}}}, \quad \forall F \in L_{t}^{1} L_{x}^{b} L_{v}^{c}, \forall G \in L_{t}^{q^{\prime}} L_{x}^{r^{\prime}} L_{v}^{r} .
$$

It would be enough to prove that

$$
B\left(\delta_{\epsilon} * f, G\right) \rightarrow \int_{-\infty}^{\infty}\langle U(t) f, G\rangle d t
$$

since we have that $\left\|\delta_{\epsilon} * f\right\|_{L_{t}^{1} L_{x}^{b} L_{v}^{c}}=\|f\|_{L_{x}^{b} L_{v}^{c}}$, for any $\epsilon>0$. To prove the limit it would be enough to consider only nonnegative functions $f$ and smooth nonnegative functions $G$ of compact support in $t \geq 0$. For $t>0$ we have

$$
\begin{aligned}
B\left(\delta_{\epsilon} f, G\right) & =\iint_{-\infty}^{t}\left\langle\delta_{\epsilon}(s) * f, U(s-t) G\right\rangle d s d t \\
& =\int_{-\infty}^{\infty} \int_{-\infty}^{\infty} \delta_{\epsilon}(t-s)\langle f, U(-s) G\rangle d s d t \rightarrow \int\langle U(t) f, G\rangle d t .
\end{aligned}
$$


The last limit is justified by the fact that the function $h(s)=\langle f, U(-s) G\rangle$ is continuous and thus $\delta_{\epsilon} * h(t) \rightarrow h(t)$ as $\epsilon \rightarrow 0$. Then, in view of Fatou's lemma,

$$
\begin{aligned}
\int\langle U(t) f, G\rangle d t & \leq \liminf _{\epsilon \rightarrow 0} B\left(\delta_{\epsilon} f, G\right) \\
& \lesssim\|f\|_{L_{x}^{b} L_{v}^{c}}\|G\|_{L_{t}^{q^{\prime}} L_{x}^{r^{\prime}} L_{v}^{p^{\prime}}}, \quad \forall f \in L_{x}^{b} L_{v}^{c} .
\end{aligned}
$$

(ii) This follows directly from Lemma 5.2 .

Remark 5.5. We shall need a slightly more general form of the the Equivalence Theorem in the sequel, where the temporal norm is the Lorentz $L^{q, s}$-norm in time. In fact, we shall be only interested in the case when $s=q$ and thus $L^{q, q}$ is equivalent to $L^{q}$, and in the cases when $s=1$ or $s=\infty$. The proof is almost identical and will be omitted.

5.3. Local in time decompositions and scaling. We shall further refine our main tool which is Lemma 5.2 by introducing a temporal localization of the bilinear form $B$. More precisely, $B$ shall be decomposed into a sum of scaling invariant dyadic pieces induced by a Whitney's dyadic decomposition applied on the domain $\Omega=\{(t, s) \mid s<t\}$ used to define $B$.

Definition 5.6. We call any positive integer that is a power of two a dyadic number. Furthermore, we call a square $Q$ in $\mathbb{R}^{2}$ dyadic if its side length is a dyadic number and the coordinates of its vertices are integer multiples of dyadic numbers.

We apply Whitney's dyadic decomposition on $\Omega$ and obtain the family $\mathcal{O}$ of essentially disjoint dyadic squares $Q$ (by that we mean that overlapping on the sides is still possible) such that the distance between any square $Q \in \mathcal{O}$ and the boundary of $\Omega(\{(t, s) \mid t=s\})$ is approximately proportional to the diameter of $Q$. This is immediately obvious in Figure 2. By $\mathcal{O}_{\lambda}$ we denote the collection of all

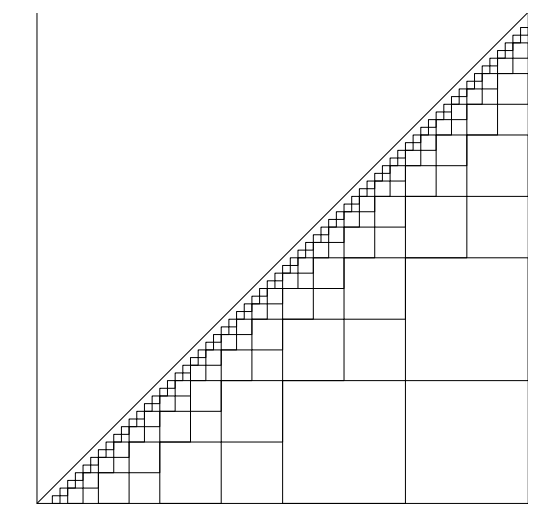

FiguRE 2. Whitney's decomposition for the region $s<t$

squares in $\mathcal{O}$ whose side length is $\lambda$. Thus we obtain the representation

$$
B(F, G)=\sum_{\lambda} \sum_{Q \in \mathcal{O}_{\lambda}} B_{Q}(F, G), \quad \text { where } \Omega=\bigcup_{\lambda} \bigcup_{Q \in \mathcal{O}_{\lambda}}
$$


and

$$
B_{Q}(F, G)=\iint_{Q}\left\langle U^{*}(s) F(s), U^{*}(t) G(t)\right\rangle d s d t .
$$

Furthermore, whenever $Q=J \times I$ and $Q \in \mathcal{O}_{\lambda}$ we have

$$
\lambda=|I|=|J| \sim \operatorname{dist}(\Omega, \partial \Omega) \sim \operatorname{dist}(I, J) .
$$

The localized bilinear operator $B_{Q}$ scales in the following way

$$
\left|B_{Q}(F, G)\right| \lesssim \lambda^{\beta(q, r, \tilde{q}, \tilde{r})}\|F\|_{L_{t}^{\tilde{q}^{\prime}}\left(J ; L_{x}^{\tilde{r}^{\prime}} L_{v}^{\tilde{p}^{\prime}}\right)}\|G\|_{L_{t}^{q^{\prime}}\left(I ; L_{x}^{r^{\prime}} L_{v}^{p^{\prime}}\right)},
$$

where

$$
\beta(q, r, \tilde{q}, \tilde{r})=\frac{1}{q}+\frac{1}{\tilde{q}}-n\left(1-\frac{1}{r}-\frac{1}{\tilde{r}}\right) .
$$

The range of the Lebesgue exponents $(1 / q, 1 / r, 1 / p, 1 / \tilde{q}, 1 / \tilde{r}, 1 / \tilde{p})$ for which we can prove that (54) holds for every $Q \in \mathcal{O}_{\lambda}$ will be presented in Lemma 7.4 Property (54) implies

Lemma 5.7. If $\frac{1}{q}+\frac{1}{\tilde{q}} \leq 1$, then

$$
\sum_{Q \in \mathcal{O}_{\lambda}}\left|B_{Q}(F, G)\right| \lesssim \lambda^{\beta(q, r, \tilde{q}, \tilde{r})}\|F\|_{L_{t}^{\tilde{q}^{\prime}}\left(\mathbb{R} ; L_{x}^{\tilde{r}^{\prime}} L_{v}^{\tilde{p}^{\prime}}\right)}\|G\|_{L_{t}^{q^{\prime}}\left(\mathbb{R} ; L_{x}^{r^{\prime}} L_{v}^{p^{\prime}}\right)} .
$$

for every $F \in L_{t}^{\tilde{q}^{\prime}}\left(\mathbb{R}, L_{x}^{\tilde{r}^{\prime}} L_{v}^{\tilde{p}^{\prime}}\right)$, and every $G \in L_{t}^{q^{\prime}}\left(\mathbb{R} ; L_{x}^{r^{\prime}} L_{v}^{p^{\prime}}\right)$.

Proof. In view of (54)

$$
\sum_{Q \in \mathcal{O}_{\lambda}}\left|B_{Q}(F, G)\right| \lesssim \lambda^{\beta(q, r, \tilde{q}, \tilde{r})} \sum_{Q \in \mathcal{O}_{\lambda}, Q=J \times I}\|F\|_{L_{t}^{\tilde{q}^{\prime}}\left(J ; L_{x}^{\tilde{r}^{\prime}} L_{v}^{\tilde{p^{\prime}}}\right)}\|G\|_{L_{t}^{q^{\prime}}\left(I ; L_{x}^{L^{\prime}} L_{v}^{p^{\prime}}\right)} .
$$

The claim now follows immediately from Lemma 5.8 below.

Lemma 5.8. Suppose $\frac{1}{p}+\frac{1}{\tilde{p}} \geq 1$. Then

$$
\sum_{Q \in \mathcal{O}_{\lambda}, Q=J \times I}\|f\|_{L^{\tilde{p}}(J)}\|g\|_{L^{p}(I)} \leq\|f\|_{L^{\tilde{p}}(\mathbb{R})}\|g\|_{L^{p}(\mathbb{R})} .
$$

Proof. The lemma follows directly from the inequality

$$
\sum_{j}\left|a_{j} b_{j}\right| \leq\left(\sum_{j}\left|a_{j}\right|^{\tilde{p}}\right)^{\frac{1}{\tilde{p}}}\left(\sum_{j}\left|b_{j}\right|^{p}\right)^{\frac{1}{p}},
$$

which holds in the range $\frac{1}{p}+\frac{1}{\tilde{p}} \geq 1$, and the fact that for each dyadic interval $I$ there are at most two dyadic squares in $\mathcal{O}_{\lambda}$ with side $I$.

We now introduce the bilinear operator $A: L_{t}^{\tilde{q}^{\prime}} L_{x}^{\tilde{r}^{\prime}} L_{v}^{\tilde{r}^{\prime}} \times L_{t}^{q^{\prime}} L_{x}^{r^{\prime}} L_{v}^{p^{\prime}} \rightarrow l_{s}^{\infty}$, (for a definition of $l_{s}^{\infty}$ see below), defined by the formula

$$
A(F, G)=\left\{b_{\lambda}\right\}_{\lambda \in 2^{\mathbb{Z}}}=\left\{\sum_{Q \in \mathcal{O}_{\lambda}}\left|B_{Q}(F, G)\right|\right\}_{\lambda \in 2^{\mathbb{Z}}} .
$$

For instance, this operator is bounded whenever we have property (54) (see Lemma 7.4), $q \geq \tilde{q}^{\prime}$, and $s=-\beta(q, r, \tilde{q}, \tilde{r})$. Clearly, the boundedness of $A: L_{t}^{\tilde{q}^{\prime}} L_{x}^{\tilde{r}^{\prime}} L_{v}^{\tilde{p}^{\prime}} \times$ 
$L_{t}^{q^{\prime}} L_{x}^{r^{\prime}} L_{v}^{p^{\prime}} \rightarrow l^{1}$ implies the boundedness of $B: L_{t}^{\tilde{q}^{\prime}} L_{x}^{\tilde{r}^{\prime}} L_{v}^{\tilde{p}^{\prime}} \times L_{t}^{q^{\prime}} L_{x}^{r^{\prime}} L_{v}^{p^{\prime}} \rightarrow \mathbb{C}$. Thus, in view of Lemma 5.2, the estimate

$$
\left\|\left\{b_{\lambda}\right\}\right\|_{l^{1}} \lesssim\|F\|_{L_{t}^{\tilde{q}^{\prime}} L_{x}^{\tilde{r}^{\prime}} L_{v}^{\tilde{p}^{\prime}}}\|G\|_{L_{t}^{q^{\prime}} L_{x}^{r^{\prime}} L_{v}^{p^{\prime}}}, \quad \forall F \in L_{t}^{\tilde{q}^{\prime}} L_{x}^{\tilde{r}^{\prime}} L_{v}^{\tilde{p}^{\prime}}, \forall G \in L_{t}^{q} L_{x}^{r} L_{v}^{p},
$$

implies the boundedness of $W(t): L_{t}^{\tilde{q}^{\prime}} L_{x}^{\tilde{r}^{\prime}} L_{v}^{\tilde{p}^{\prime}} \rightarrow L_{t}^{q} L_{x}^{r} L_{v}^{p}$. We summarize this fact in

Lemma 5.9. The boundedness of the bilinear operator $A: L_{t}^{\tilde{q}^{\prime}} L_{x}^{\tilde{r}^{\prime}} L_{v}^{\tilde{p}^{\prime}} \times L_{t}^{q^{\prime}} L_{x}^{r^{\prime}} L_{v}^{p^{\prime}} \rightarrow$ $l^{1}$ implies the inhomogeneous Strichartz estimate

$$
\|W(t) F\|_{L_{t}^{q} L_{x}^{r} L_{v}^{p}} \lesssim\|F\|_{L_{t}^{\tilde{q}^{\prime}} L_{x}^{\tilde{r}^{\prime}} L_{v}^{\tilde{p}^{\prime}}}, \quad \forall F \in L_{t}^{\tilde{q}^{\prime}} L_{x}^{\tilde{r}^{\prime}} L_{v}^{\tilde{p}^{\prime}}
$$

In our proofs of the inhomogeneous Strichartz estimates for the KT equation we shall be making a repeated use of this lemma. We shall also need a number of standard results from the theory of Interpolation Spaces which are given in the remaining part of this paragraph. By $L^{p}=L^{p}(X ; \mathcal{B})$ and $L^{p, q}=L^{p, q}(X ; \mathcal{B})$ we denote the Lebesgue space and the Lorentz space respectively of vector-valued functions that map a fixed measure space $(X, d \mu)$ to a fixed Banach space $\mathcal{B}$.

Lemma 5.10 (see [3, p. 113]). Suppose that $0<p_{0}, p_{1}, q_{0}, q_{1} \leq \infty, 0<\theta<1$, and $p_{0} \neq p_{1}$. Then

$$
\left(L^{p_{0}, q_{0}}, L^{p_{1}, q_{1}}\right)_{\theta, q}=L^{p, q},
$$

where $1 / p=(1-\theta) / p_{0}+\theta / p_{1}$.

Suppose that $\mathcal{B}_{0}$ and $\mathcal{B}_{1}$ are two Banach spaces that are compatible for interpolation.

Lemma 5.11 (see the Appendix of [6]). For every $1 \leq p_{0}, p_{1}<\infty, 0<\theta<1$, $1 / p=(1-\theta) / p_{0}+\theta / p_{1}$ and $p \leq q$ we have

$$
L^{p}\left(X ;\left(\mathcal{B}_{0}, \mathcal{B}_{1}\right)_{\theta, q}\right) \hookrightarrow\left(L^{p_{0}}\left(X ; \mathcal{B}_{0}\right), L^{p_{1}}\left(X ; \mathcal{B}_{1}\right)\right)_{\theta, q} .
$$

Denote by $l_{s}^{p}$ the space of number sequences with a norm

$$
\begin{aligned}
\left\|\{a\}_{j \in \mathbb{Z}}\right\|_{l_{s}^{p}} & =\left(2^{j s}\left|a_{j}\right|^{p}\right)^{1 / p}, & & 1 \leq p<\infty, \\
\left\|\{a\}_{j \in \mathbb{Z}}\right\|_{l_{s}^{\infty}} & =\sup _{j \in \mathbb{Z}} 2^{j s}\left|a_{j}\right|, & & p=\infty .
\end{aligned}
$$

Lemma 5.12 (See Theorem 5.6.1 in [3]). We have the identity

$$
\left(l_{s_{0}}^{\infty}, l_{s_{1}}^{\infty}\right)_{\theta, 1}=l_{s}^{1},
$$

where $s_{0}, s_{1} \in \mathbb{R}, s_{0} \neq s_{1}$ and $s=(1-\theta) s_{0}+\theta s_{1}$.

Lemma 5.13 (See p. 76 in 3]). Suppose that $\left(\mathcal{A}_{0}, \mathcal{A}_{1}\right),\left(\mathcal{B}_{0}, \mathcal{B}_{1}\right)$, and $\left(\mathcal{C}_{0}, \mathcal{C}_{1}\right)$ are interpolation couples and that the bilinear operator $T$ acts as a bounded transformation as indicated below:

$$
\begin{aligned}
& T: \mathcal{A}_{0} \times \mathcal{B}_{0} \rightarrow \mathcal{C}_{0}, \\
& T: \mathcal{A}_{1} \times \mathcal{B}_{1} \rightarrow \mathcal{C}_{1} .
\end{aligned}
$$

If $\theta_{0} \in(0,1), p, q, r \in[1, \infty]$, and $1+1 / r=1 / p+1 / q$, then $T$ also acts as a bounded transformation in the following way:

$$
T:\left(\mathcal{A}_{0}, \mathcal{A}_{1}\right)_{\theta, p} \times\left(\mathcal{B}_{0}, \mathcal{B}_{1}\right)_{\theta, q} \rightarrow\left(\mathcal{C}_{0}, \mathcal{C}_{1}\right)_{\theta, r} .
$$


Lemma 5.14 (See pp. 76-77 in [3]). Suppose that $\left(\mathcal{A}_{0}, \mathcal{A}_{1}\right),\left(\mathcal{B}_{0}, \mathcal{B}_{1}\right)$, and $\left(\mathcal{C}_{0}, \mathcal{C}_{1}\right)$ are interpolation couples and that the bilinear operator $T$ acts as a bounded transformation as indicated below:

$$
\begin{aligned}
& T: \mathcal{A}_{0} \times \mathcal{B}_{0} \rightarrow \mathcal{C}_{0}, \\
& T: \mathcal{A}_{0} \times \mathcal{B}_{1} \rightarrow \mathcal{C}_{1}, \\
& T: \mathcal{A}_{1} \times \mathcal{B}_{0} \rightarrow \mathcal{C}_{1} .
\end{aligned}
$$

If $\theta_{0}, \theta_{1} \in(0,1)$ and $p, q, r \in[1, \infty]$ are such that $1 / p+1 / q \geq 1$, then $T$ also acts as a bounded transformation in the following way:

$$
T:\left(\mathcal{A}_{0}, \mathcal{A}_{1}\right)_{\theta_{0}, p r} \times\left(\mathcal{B}_{0}, \mathcal{B}_{1}\right)_{\theta_{1}, q r} \rightarrow\left(\mathcal{C}_{0}, \mathcal{C}_{1}\right)_{\theta_{0}+\theta_{1}, r} .
$$

\section{Proof of Strichartz estimates for admissible exponents}

In this paragraph we prove only the validity of the estimates in Theorem 2.4 , The investigation of their sharpness shall be made in Section 9 by means of counterexamples.

Our plan is the following one. We shall first prove the homogeneous estimate

$$
\|U(t) f\|_{L_{t}^{q} L_{x}^{r} L_{v}^{r^{\prime}}} \lesssim\|f\|_{L_{x}^{2}}
$$

via the corresponding estimate for the $T T^{*}$-operator for non-endpoint exponent triplets. Then in view of the invariance (34) we obtain

$$
\|U(t) f\|_{L_{t}^{q} L_{x}^{r} L_{v}^{p}} \lesssim\|f\|_{L_{x}^{a}}, \quad 1 \leq a \leq \infty, q>a .
$$

By duality and composition this implies the estimate

$$
\|W(t) F\|_{L_{t}^{q} L_{x}^{r} L_{v}^{p}} \lesssim\|F\|_{L_{t}^{\tilde{q}^{\prime}} L_{x}^{\tilde{r}^{\prime}} L_{v}^{\tilde{p}^{\prime}}}
$$

whenever $a=\operatorname{HM}(p, r)=\operatorname{HM}\left(\tilde{p}^{\prime}, \tilde{r}^{\prime}\right)$ and $(\tilde{q}, \tilde{r}, \tilde{p})$ is another non-endpoint KTadmissible exponent triplet.

Proof. In view of the decay estimate

$$
\|U(t) f\|_{L_{x}^{r} L_{v}^{r^{\prime}}} \lesssim \frac{1}{|t|^{\beta(r)}}\|f\|_{L_{x}^{r^{\prime} L_{v}^{r}}}, \quad 2 \leq r \leq \infty,
$$

where $\beta(r)=n(1-2 / r)$, we have

$$
\left\|T T^{*} F\right\|_{L_{x}^{r} L_{v}^{r^{\prime}}} \lesssim \int_{-\infty}^{\infty}\|U(t-s) F(s)\|_{L_{x}^{r} L_{v}^{r^{\prime}}} d s \lesssim \int_{-\infty}^{\infty} \frac{\|F(s)\|_{L_{x}^{r^{\prime}} L_{v}^{r}}}{|t-s|^{\beta(r)}} d s .
$$

We take the $L^{q}$-norm in $t$ and in view of the Hardy-Littlewood-Sobolev (HLS) theorem of fractional integration, see [2, pp. 228-229], [15, we obtain

$$
\left\|T T^{*} F\right\|_{L_{t}^{q} L_{x}^{r} L_{v}^{r^{\prime}}} \lesssim\|F\|_{L_{t}^{q^{\prime}} L_{x}^{r^{\prime}} L_{v}^{r}},
$$

whenever $0<\beta(r)<1,1+1 / q=1 / q^{\prime}+\beta(r)$. The latter conditions are equivalent to $2<r<r^{*}(2), 1 / q+n / r=n / 2$. The left endpoint $r=2$ follows trivially from the transport estimate (40).

The right endpoint $r=r^{*}(2)$ remains unresolved in the context of the KT equation, unlike that of the wave and the Schrödinger equations, where it has been resolved (in the positive) by Keel and Tao [11] (1997). In the setting of the inhomogeneous estimates, the "double endpoint" for which both exponent triplets are endpoint remains unresolved. However, if $(q, r, p)$ is non-endpoint and $(\tilde{q}, \tilde{r}, \tilde{p})$ 
is endpoint the corresponding inhomogeneous estimate is still non-endpoint (since in such case $q>\tilde{q}^{\prime}$ ) and thus holds true in view of Theorem 2.5 whose proof will be given in Section 8 .

\section{LOCAL INHOMOGENEOUS ESTIMATES}

In order to go beyond the "standard" Strichartz estimates for the KT equation proved in the previous section we shall adapt and apply techniques pioneered by Foschi [7, and Keel and Tao [1]. We have also considered the works by Vilela [17] and Taggart 16.

Our goal is to find the maximal range of estimates where we have the scaling property

$$
\left\|W(t)\left[\chi_{\lambda J} F\right]\right\|_{L^{q}\left(\lambda I ; L_{x}^{r} L_{v}^{p}\right)} \lesssim \lambda^{\frac{1}{q}+\frac{1}{\tilde{q}}-n\left(1-\frac{1}{r}-\frac{1}{\bar{r}}\right)}\|F\|_{L_{\tilde{q}^{\prime}}\left(\lambda J ; L_{x}^{\tilde{r}^{\prime}} L_{v}^{\tilde{p}^{\prime}}\right)}, \quad \forall \lambda>0,
$$

for any two unit intervals $I$ and $J$ separated by a unit distance and any $F \in$ $L_{t}^{\tilde{q}^{\prime}}\left(\mathbb{R}, L_{x}^{\tilde{r}^{\prime}} L_{v}^{\tilde{p}^{\prime}}\right)$, where $\chi_{\lambda J}$ denotes the characteristic function of the rescaled interval $\lambda J$. Note that (57) is equivalent to (54).

Lemma 7.1. Estimate (57) holds for any two non-endpoint KT-admissible triplets $(q, r, p)$ and $(\tilde{q}, \tilde{r}, \tilde{p})$ with $a=\tilde{a}^{\prime}$.

Proof. The proof follows trivially from Theorem 2.4 due to the fact that $\beta(q, r, \tilde{q}, \tilde{r})$ $=0$ under the hypothesis of the lemma.

Lemma 7.2. Estimate (57) holds with $(q, r, p)=(\infty, r, p)$ and $(\tilde{q}, \tilde{r}, \tilde{p})=\left(\infty, p^{\prime}, r^{\prime}\right)$, where $1 \leq p \leq r \leq \infty$.

Proof. Due to the decay estimate (41) we have that

$$
\begin{aligned}
\sup _{t \in \lambda I}\left\|W(t)\left[\chi_{\lambda J} F\right]\right\|_{L_{x}^{r} L_{v}^{p}} & \lesssim \sup _{t \in \lambda I} \int_{\lambda J} \frac{\|F(\tau)\|_{L_{x}^{p} L_{v}^{r}}}{|t-\tau|^{n\left(\frac{1}{p}-\frac{1}{r}\right)}} d \tau \\
& \lesssim \lambda^{\beta\left(\infty, r, \infty, p^{\prime}\right)}\|F\|_{L^{1}\left(\lambda J ; L_{x}^{p} L_{v}^{r}\right)} .
\end{aligned}
$$

Lemma 7.3. Whenever $(q, r, p)$ and $(\tilde{q}, \tilde{r}, \tilde{p})$ are exponent triplets for which estimate (57) holds, we have that (57) also holds with $(Q, r, p)$ and $(\tilde{Q}, \tilde{r}, \tilde{p})$, where $1 \leq Q \leq q, 1 \leq \tilde{Q} \leq \tilde{q}$.

Proof. A trivial application of Hölder's inequality

$$
\begin{aligned}
& \left\|W(t)\left[\chi_{\lambda J} F\right]\right\|_{L^{Q}\left(\lambda I ; L_{x}^{r} L_{v}^{p}\right)} \lesssim \lambda^{\frac{1}{Q}-\frac{1}{q}}\left\|W(t)\left[\chi_{\lambda J} F\right]\right\|_{L^{q}\left(\lambda I ; L_{x}^{r} L_{v}^{p}\right)} \\
& \quad \lesssim \lambda^{\beta(Q, r, \tilde{q}, \tilde{r})}\|F\|_{L^{\tilde{q}^{\prime}}\left(\lambda J ; L_{x}^{\tilde{r}^{\prime}} L_{v}^{\tilde{p}^{\prime}}\right)} \lesssim \lambda^{\beta(Q, r, \tilde{Q}, \tilde{r})}\|F\|_{L^{\tilde{Q}^{\prime}\left(\lambda J ; L_{x}^{\tilde{r}^{\prime}} L_{v}^{\tilde{p}^{\prime}}\right)}}
\end{aligned}
$$

Let us define the range of validity of the local estimates (57) as the set $\mathcal{E} \subset \mathbb{R}^{6}$ consisting of exponent vectors $(1 / q, 1 / r, 1 / p, 1 / \tilde{q}, 1 / \tilde{r}, 1 / \tilde{p})$ in $\mathbb{R}^{6}$ that correspond to valid estimates (57). We shall only find the convex hull $\mathcal{E}_{0} \subseteq \mathcal{E}$ of the points in $\mathbb{R}^{6}$ that correspond to the estimates in the three lemmas above. The question whether $\mathcal{E}_{0}=\mathcal{E}$ remains open. 
Lemma 7.4 (Local inhomogeneous estimates). Estimate (57) holds whenever the exponent triplets $(q, r, p),(\tilde{q}, \tilde{r}, \tilde{p})$ satisfy the following conditions

$$
\begin{gathered}
0 \leq \frac{1}{q}, \frac{1}{\tilde{q}} \leq 1, \quad 0<\frac{1}{p}, \frac{1}{\tilde{p}}, \frac{1}{r}, \frac{1}{\tilde{r}} \leq 1, \\
\frac{1}{r} \leq \frac{1}{p}, \quad \frac{1}{\tilde{r}} \leq \frac{1}{\tilde{p}}, \quad \operatorname{HM}(p, r)=\operatorname{HM}\left(\tilde{p}^{\prime}, \tilde{r}^{\prime}\right), \\
\frac{1}{\tilde{r}}-\frac{1}{\tilde{p}}-\frac{1}{r}+\frac{1}{p} \leq \frac{2}{n q}, \quad \frac{1}{r}-\frac{1}{p}-\frac{1}{\tilde{r}}+\frac{1}{\tilde{p}} \leq \frac{2}{n \tilde{q}}, \\
\frac{n-1}{p^{\prime}}<\frac{n}{\tilde{r}}, \quad \frac{n-1}{\tilde{p}^{\prime}}<\frac{n}{r},
\end{gathered}
$$

or if the point $(1 / q, 1 / r, 1 / p, 1 / \tilde{q}, 1 / \tilde{r}, 1 / \tilde{p})$ lies inside one of the "cubic" sets in $\mathbb{R}^{6}$ below

$$
\begin{array}{ll}
(\kappa, 0, \mu, \nu, 1-\mu, 1), & 0 \leq \kappa, \mu, \nu \leq 1, \\
(\kappa, 1-\mu, 1, \nu, 0, \mu), & 0 \leq \kappa, \mu, \nu \leq 1 .
\end{array}
$$

Proof. We apply the Riesz-Thorin convexity theorem to interpolate between the already proven local estimates. To that end we need to find the convex hull of the sets in $\mathbb{R}^{6}$ associated with Lemmas 7.1 and 7.2 and then expand that set by the rule given in Lemma 7.3

The range of validity $S_{1}$ of the local estimates in Lemma 7.1 is given by the system

$$
\begin{gathered}
0<\frac{1}{r}, \frac{1}{\tilde{r}} \leq 1, \quad 0 \leq \frac{1}{q}, \frac{1}{\tilde{q}}, \frac{1}{p}, \frac{1}{\tilde{p}} \leq 1, \\
\frac{1}{q}=\frac{n}{2}\left(\frac{1}{p}-\frac{1}{r}\right), \quad \frac{1}{\tilde{q}}=\frac{n}{2}\left(\frac{1}{\tilde{p}}-\frac{1}{\tilde{r}}\right), \\
\frac{1}{r}+\frac{1}{p}+\frac{1}{\tilde{r}}+\frac{1}{\tilde{p}}=2, \\
\frac{n-1}{p}<\frac{n+1}{r}, \quad \frac{n-1}{\tilde{p}}<\frac{n+1}{\tilde{r}},
\end{gathered}
$$

or if $(1 / q, 1 / r, 1 / p, 1 / \tilde{q}, 1 / \tilde{r}, 1 / \tilde{p}) \in\{B=(0,0,0,0,1,1), C=(0,1,1,0,0,0)\}$.

Note that $S_{1}$ is a convex polyhedron in $\mathbb{R}^{6}$ and the two points $B$ and $C$ lie on its boundary. The range of validity $S_{2}$ of the local estimates in Lemma 7.2 is the convex hull, in fact a triangle, of the three points

$$
A=(0,0,1,0,0,1), \quad B=(0,0,0,0,1,1), \quad C=(0,1,1,0,0,0) .
$$

The vertices $B$ and $C$ are already included in $S_{1}$ and thus it would suffice to take only the vertex $A$. Hence, we obtain the following set

$$
\begin{array}{llll}
\frac{1}{Q}=\frac{\theta}{q}, & \frac{1}{R}=\frac{\theta}{r}, & \frac{1}{P}=1-\theta+\frac{\theta}{p}, & \\
\frac{1}{\tilde{Q}}=\frac{\theta}{\tilde{q}}, & \frac{1}{\tilde{R}}=\frac{\theta}{\tilde{r}}, & \frac{1}{\tilde{P}}=1-\theta+\frac{\theta}{\tilde{p}}, & 0 \leq \theta \leq 1,
\end{array}
$$

where $(1 / Q, 1 / R, 1 / P, 1 / \tilde{Q}, 1 / \tilde{R}, 1 / \tilde{P})$ are the coordinates of the new set $S_{3}$ written in terms of $(1 / q, 1 / r, 1 / p, 1 / \tilde{q}, 1 / \tilde{r}, 1 / \tilde{p})$ and $\theta$. Of course, we must also add to $S_{3}$ the line segments $[A, B]$ and $[A, C]$. We shall treat this case separately at the end. 
Finally, we apply the rule given in Lemma 7.3 and thus we replace the equations for $Q$ and $\tilde{Q}$ above with the following inequalities

$$
1 \geq \frac{1}{Q} \geq \frac{\theta}{q}, \quad 1 \geq \frac{1}{\tilde{Q}} \geq \frac{\theta}{\tilde{q}},
$$

plus the restrictions

$$
\frac{1}{r} \leq \frac{1}{p}, \quad \frac{1}{\tilde{r}} \leq \frac{1}{\tilde{p}},
$$

which were implicitly assumed in (64).

1. We first eliminate $q$ and $\tilde{q}$ from the system for $S_{1}$ to obtain

$$
\begin{aligned}
\frac{1}{Q} \geq \frac{n}{2}\left(\frac{\theta}{p}-\frac{\theta}{r}\right), & \Leftrightarrow \quad \frac{1}{Q} \geq \frac{n}{2}\left(\theta-1+\frac{1}{P}-\frac{1}{R}\right), \\
& \Leftrightarrow \quad \theta \leq \frac{1}{P^{\prime}}+\frac{1}{R}+\frac{2}{n Q} .
\end{aligned}
$$

Similarly,

$$
\theta \leq \frac{1}{\tilde{P}^{\prime}}+\frac{1}{\tilde{R}}+\frac{2}{n \tilde{Q}}, \quad \frac{1}{Q}, \frac{1}{\tilde{Q}} \leq 1 .
$$

2. As expected, condition (65) is invariant

$$
\frac{1}{R}+\frac{1}{P}+\frac{1}{\tilde{R}}+\frac{1}{\tilde{P}}=2 .
$$

3. Reworking condition (66), we obtain

$$
\theta<\frac{n+1}{n-1} \frac{1}{R}+\frac{1}{P^{\prime}}, \quad \theta<\frac{n+1}{n-1} \frac{1}{\tilde{R}}+\frac{1}{\tilde{P}^{\prime}} .
$$

4. Condition (68) is replaced by

$$
\frac{1}{P^{\prime}}+\frac{1}{R} \leq \theta, \quad \frac{1}{\tilde{P}^{\prime}}+\frac{1}{\tilde{R}} \leq \theta .
$$

5. Finally, conditions (63) are transformed into

$$
\frac{1}{P^{\prime}}, \frac{1}{\tilde{P}^{\prime}}, \frac{1}{R}, \frac{1}{\tilde{R}} \leq \theta, \quad 0 \leq \frac{1}{Q}, \frac{1}{\tilde{Q}}, \frac{1}{P}, \frac{1}{\tilde{P}}, \frac{1}{R}, \frac{1}{\tilde{R}} \leq 1 .
$$

6. We group all conditions obtained in the previous 5 steps according to their type

$$
\begin{gathered}
0, \frac{1}{P^{\prime}}, \frac{1}{\tilde{P}^{\prime}}, \frac{1}{R}, \frac{1}{\tilde{R}}, \frac{1}{P^{\prime}}+\frac{1}{R}, \frac{1}{\tilde{P}^{\prime}}+\frac{1}{\tilde{R}} \leq \theta . \\
\theta \leq \frac{1}{R}+\frac{1}{P^{\prime}}+\frac{2}{n Q}, \frac{1}{\tilde{R}}+\frac{1}{\tilde{P}^{\prime}}+\frac{2}{n \tilde{Q}}, \frac{n+1}{n-1} \frac{1}{R}+\frac{1}{P^{\prime}}, \frac{n+1}{n-1} \frac{1}{\tilde{R}}+\frac{1}{\tilde{P}^{\prime}}, 1 . \\
0 \leq \frac{1}{Q}, \frac{1}{\tilde{Q}}, \frac{1}{P}, \frac{1}{\tilde{P}}, \frac{1}{R}, \frac{1}{\tilde{R}} \leq 1, \quad \frac{1}{P}+\frac{1}{R}+\frac{1}{\tilde{R}}+\frac{1}{\tilde{P}}=2 .
\end{gathered}
$$

7. We discard the redundant conditions like

$$
0, \frac{1}{P^{\prime}}, \frac{1}{\tilde{P}^{\prime}}, \frac{1}{R}, \frac{1}{\tilde{R}} \leq \theta,
$$

which are all weaker than the other two in (699).

There exists $\theta$ solving all inequalities in (69), (70), if and only if every quantity in (69) is bounded from above by any quantity in (70). Thus we form all possible 
combinations between the quantities in the two types of (reduced) inequalities to obtain the following set of conditions

$$
\begin{aligned}
\frac{1}{R}+\frac{1}{P^{\prime}} \leq \frac{1}{\tilde{R}}+\frac{1}{\tilde{P}^{\prime}}+\frac{2}{n \tilde{Q}}, & \frac{1}{\tilde{R}}+\frac{1}{\tilde{P}^{\prime}} \leq \frac{1}{R}+\frac{1}{P^{\prime}}+\frac{2}{n Q}, \\
\frac{1}{R}+\frac{1}{P^{\prime}}<\frac{n+1}{n-1} \frac{1}{\tilde{R}}+\frac{1}{\tilde{P}^{\prime}}, & \Leftrightarrow \quad \frac{n-1}{P^{\prime}}<\frac{n}{\tilde{R}} \\
\frac{1}{\tilde{R}}+\frac{1}{\tilde{P}^{\prime}}<\frac{n+1}{n-1} \frac{1}{R}+\frac{1}{P^{\prime}}, & \Leftrightarrow \frac{n-1}{\tilde{P}^{\prime}}<\frac{n}{R}, \\
\frac{1}{R} \leq \frac{1}{P}, & \frac{1}{\tilde{R}} \leq \frac{1}{\tilde{P}},
\end{aligned}
$$

describing the region $S_{3}$.

8. We apply the rule given in Lemma 7.3 to the two line segments $[A, B]$ and $[A, C]$ to obtain the following two "cubic" regions in $\mathbb{R}^{6}$

$$
\begin{array}{ll}
(\mu, 0, \kappa, \nu, 1-\kappa, 1), & 0 \leq \mu, \nu, \kappa \leq 1, \\
(\mu, 1-\kappa, 1, \nu, 0, \kappa), & 0 \leq \mu, \nu, \kappa \leq 1 .
\end{array}
$$

Hence, the computation of the set $\mathcal{E}_{0}$ is finished.

\section{Proof of the generalized inhomogeneous Strichartz estimates}

8.1. Generalized inhomogeneous non-endpoint estimates. In this paragraph we prove the inhomogeneous Strichartz estimate

$$
\|W(t) F\|_{L_{t}^{q} L_{x}^{r} L_{v}^{p}} \lesssim\|F\|_{L_{t}^{\tilde{q}^{\prime}} L_{x}^{\tilde{r}^{\prime}} L_{v}^{\tilde{p}^{\prime}}}, \quad \forall F \in L_{t}^{\tilde{q}^{\prime}} L_{x}^{\tilde{r}^{\prime}} L_{v}^{\tilde{p}^{\prime}},
$$

in the range $q>\tilde{q}^{\prime}$. Thanks to Lemma [5.9, we have reduced this problem to showing the estimate

$$
\left\|\left\{b_{\lambda}\right\}\right\|_{l^{1}} \lesssim\|F\|_{L_{t}^{\tilde{q}^{\prime}} L_{x}^{\tilde{r}^{\prime}} L_{v}^{\tilde{p}^{\prime}}}\|G\|_{L_{t}^{q^{\prime}} L_{x}^{r^{\prime}} L_{v}^{p^{p^{\prime}}}}, \quad \forall F \in L_{t}^{\tilde{q}^{\prime}} L_{x}^{\tilde{r}^{\prime}} L_{v}^{\tilde{p}^{\prime}}, \forall G \in L_{t}^{q^{\prime}} L_{x}^{r^{\prime}} L_{v}^{p^{\prime}},
$$

where

$$
\left\{b_{\lambda}\right\}_{\lambda \in 2^{\mathbb{Z}}}=\left\{\sum_{Q \in \mathcal{O}_{\lambda}}\left|B_{Q}(F, G)\right|\right\}_{\lambda \in 2^{\mathbb{Z}}} .
$$

We shall next specify the range of validity of the above estimates in terms of the vector

$$
P=(1 / q, 1 / r, 1 / p, 1 / \tilde{q}, 1 / \tilde{r}, 1 / \tilde{p}) \in \mathcal{E}_{0}
$$

for which

$$
1 / q+1 / \tilde{q}=n(1-1 / r-1 / \tilde{r}) .
$$

Let us denote by $\Delta$ the set in $\mathbb{R}^{2}$

$$
\left\{(x, y) \mid x>0, y>0, x+y<1,(x, 1 / r, 1 / p, y, 1 / \tilde{r}, 1 / \tilde{p}) \in \mathcal{E}_{0}\right\}
$$

and its interior (largest open subset) by $\operatorname{int}(\Delta)$. In this paragraph we prove that for any such vector $P \in \operatorname{int}(\Delta)$ the corresponding inhomogeneous Strichartz estimate holds true.

Under the assumption of the latter condition and in view of Corollary [5.7 we have the estimate

$$
\left|b_{\lambda}\right| \lesssim \lambda^{\beta(q, r, \tilde{q}, \tilde{r})}\|F\|_{L_{t}^{\tilde{q}^{\prime}} L_{x}^{\tilde{r}^{\prime}} L_{v}^{\tilde{p}^{\prime}}}\|G\|_{L_{t}^{q^{\prime}} L_{x}^{r^{\prime}} L_{v}^{p^{\prime}}}
$$


or equivalently, $\left\{b_{\lambda}\right\} \in l_{s}^{\infty}$ with $s=-\beta(q, r, \tilde{q}, \tilde{r})$. Let us set

$$
1 / q_{0}=1 / q+\epsilon, \quad 1 / \tilde{q}_{0}=1 / \tilde{q}+\epsilon, \quad 1 / q_{1}=1 / q-3 \epsilon, \quad 1 / \tilde{q}_{1}=1 / \tilde{q}-3 \epsilon,
$$

for some small enough $\epsilon>0$, whose existence is guaranteed by our assumptions, such that the perturbed exponent vectors do not leave int $(\Delta)$. Then we have that $\beta\left(q_{0}, r, \tilde{q}_{0}, \tilde{r}\right)=2 \epsilon$, and $\beta\left(q_{1}, r, \tilde{q}_{0}, \tilde{r}\right)=\beta\left(q_{0}, r, \tilde{q}_{1}, \tilde{r}\right)=-2 \epsilon$. The following bilinear maps

$$
\begin{aligned}
& A: L_{t}^{\tilde{q}_{0}^{\prime}} L_{x}^{\tilde{r}^{\prime}} L_{v}^{\tilde{p}^{\prime}} \times L_{t}^{q_{0}^{\prime}} L_{x}^{r^{\prime}} L_{v}^{p^{\prime}} \rightarrow l_{-2 \epsilon}^{\infty}, \\
& A: L_{t}^{\tilde{q}_{0}^{\prime}} L_{x}^{\tilde{r}^{\prime}} L_{v}^{\tilde{p}^{\prime}} \times L_{t}^{q_{1}^{\prime}} L_{x}^{r^{\prime}} L_{v}^{p^{\prime}} \rightarrow l_{2 \epsilon}^{\infty}, \\
& A: L_{t}^{\tilde{q}_{1}^{\prime}} L_{x}^{\tilde{r}^{\prime}} L_{v}^{\tilde{p}^{\prime}} \times L_{t}^{q_{0}^{\prime}} L_{x}^{r^{\prime}} L_{v}^{p^{\prime}} \rightarrow l_{2 \epsilon}^{\infty},
\end{aligned}
$$

are bounded. In virtue of Lemma 5.14, we have that the map

$$
A:\left(L_{t}^{\tilde{q}_{0}^{\prime}} L_{x}^{\tilde{r}^{\prime}} L_{v}^{\tilde{p}^{\prime}}, L_{t}^{\tilde{q}_{1}^{\prime}} L_{x}^{\tilde{r}^{\prime}} L_{v}^{\tilde{p}^{\prime}}\right)_{1 / 4, \tilde{q}^{\prime}} \times\left(L_{t}^{q_{0}^{\prime}} L_{x}^{r^{\prime}} L_{v}^{p^{\prime}}, L_{t}^{q_{1}^{\prime}} L_{x}^{r^{\prime}} L_{v}^{p^{\prime}}\right)_{1 / 4, q^{\prime}} \rightarrow\left(l_{2 \epsilon}^{\infty}, l_{-2 \epsilon}^{\infty}\right)_{1 / 2,1}
$$

is also bounded. Finally, in view of Lemma 5.12 and the embeddings of the Lorentz spaces, we obtain

$$
A: L_{t}^{\tilde{q}^{\prime}} L_{x}^{\tilde{r}^{\prime}} L_{v}^{\tilde{p}^{\prime}} \times L_{t}^{q^{\prime}} L_{x}^{r^{\prime}} L_{v}^{p^{\prime}} \rightarrow l^{1} .
$$

All assumption made in this paragraph are explicitly stated in Theorem 2.5. part (i). We remark that condition (60) together with (73) is equivalent to $(q, r, p)$ and $(\tilde{q}, \tilde{r}, \tilde{p})$ being KT-acceptable. Furthermore, in this case the inequalities in (60) have to be taken as strict inequalities so that $P \in \operatorname{int}(\Delta)$. Let us also note that the two locally acceptable "cubic" sets in (72) give rise to the two globally acceptable "cubic cross section" sets $\Sigma_{1}$ and $\Sigma_{2}$ in Definition 2.3 .

8.2. Global inhomogeneous endpoint estimates with $\tilde{q}=\infty$. In this paragraph we prove the inhomogeneous Strichartz estimates with $P$ lying on either of the two catheti of $\triangle O A B$ in Figure 1. Since by duality both type of estimates are equivalent, it is enough to consider only the case $\tilde{q}=\infty$. We exclude the two endpoints $(0,0)$ and $(1,0)$ from our considerations. We suppose that

$$
P(1 / q, 1 / r, 1 / p, 0,1 / \tilde{r}, 1 / \tilde{p}) \in\{0<1 / q<1\} \cap \mathcal{E}_{0}
$$

and is such that $q$ satisfies every inequality of $\mathcal{E}_{0}$ as a strict inequality. We also assume the scaling condition (73) from the previous paragraph. Then we have

$$
\begin{aligned}
& A: L_{t}^{1} L_{x}^{\tilde{r}^{\prime}} L_{v}^{\tilde{p}^{\prime}} \times L_{t}^{q_{0}^{\prime}} L_{x}^{r^{\prime}} L_{v}^{p^{\prime}} \rightarrow l_{\epsilon}^{\infty}, \\
& A: L_{t}^{1} L_{x}^{\tilde{r}^{\prime}} L_{v}^{\tilde{p}^{\prime}} \times L_{t}^{q_{1}^{\prime}} L_{x}^{r^{\prime}} L_{v}^{p^{\prime}} \rightarrow l_{-\epsilon}^{\infty}
\end{aligned}
$$

where

$$
\frac{1}{q_{0}}=\frac{1}{q}-\frac{1}{\epsilon}, \quad \frac{1}{q_{1}}=\frac{1}{q}+\frac{1}{\epsilon} .
$$

The real method with parameters $(\theta, q)=(1 / 2,1)$, see Lemma [5.13] gives that

$$
A: L_{t}^{1} L_{x}^{\tilde{r}^{\prime}} L_{v}^{\tilde{p}^{\prime}} \times L_{t}^{q^{\prime}, 1} L_{x}^{r^{\prime}} L_{v}^{p^{\prime}} \rightarrow l^{1} .
$$

Equivalently, in view of the $T T^{*}$-principle,

$$
\|W(t) F\|_{L_{t}^{q, \infty} L_{x}^{r} L_{v}^{p}} \lesssim\|F\|_{L_{t}^{1} L_{x}^{\tilde{r}^{\prime}} L_{v}^{\tilde{p}^{\prime}}}
$$


for all $F \in L_{t}^{1} L_{x}^{\tilde{r}^{\prime}} L_{v}^{\tilde{r}^{\prime}}$. The explicit restrictions on the Lebesgue exponents $(q, r, p)$ and $(\infty, \tilde{r}, \tilde{p})$ are stated in Theorem 2.5 . part (ii). Analogously, the dual case is stated as part (iii) of that theorem.

In the remainder of this paragraph we address (i) the corresponding homogeneous estimates to (74) via the Equivalence Theorem 2.8 in its stronger form for Lorentz spaces and (ii) the sharpening of (74) to the Lebesgue norm $L_{t}^{q}$.

Lemma 8.1. The estimate

$$
\|U(t) f\|_{L_{t}^{q, \infty} L_{x}^{r} L_{v}^{p}} \lesssim\|f\|_{L_{x}^{b} L_{v}^{c}},
$$

holds for all $f \in L_{x}^{b} L_{v}^{c}$, whenever

$$
\begin{aligned}
& \frac{1}{q}+\frac{n}{r}=\frac{n}{b}, \quad \operatorname{HM}(r, p)=\operatorname{HM}(b, c) \stackrel{\text { def }}{=} a, \quad r<\frac{n c}{n-1}, \\
& p<b \leq a \leq c<r, \quad 1<q<\infty, 1 \leq p, \tilde{p}, r, \tilde{r}<\infty .
\end{aligned}
$$

Proof. The range of validity of estimate (75) is determined in the following way. We first write the conditions defining the set $\mathcal{E}_{0}$, however, any inequality where $q$ appears is taken as a strict inequality. To that system we add the scaling condition (73). Thus, we have that $1 / q=n(1-1 / r-1 / \tilde{r})$, and

$$
\begin{gathered}
0<\frac{1}{q}, \frac{1}{\tilde{q}}<1, \quad 0<\frac{1}{p}, \frac{1}{\tilde{p}}, \frac{1}{r}, \frac{1}{\tilde{r}} \leq 1, \\
\frac{1}{r} \leq \frac{1}{p}, \quad \frac{1}{\tilde{r}} \leq \frac{1}{\tilde{p}}, \quad \operatorname{HM}(p, r)=\operatorname{HM}\left(\tilde{p}^{\prime}, \tilde{r}^{\prime}\right), \\
\frac{1}{q}<n\left(\frac{1}{p}-\frac{1}{r}\right), \quad 0 \leq n\left(\frac{1}{\tilde{p}}-\frac{1}{\tilde{r}}\right), \\
\frac{n-1}{p^{\prime}}<\frac{n}{\tilde{r}}, \quad \frac{n-1}{\tilde{p}^{\prime}}<\frac{n}{r},
\end{gathered}
$$

or that the point $(1 / q, 1 / r, 1 / p, 0,1 / \tilde{r}, 1 / \tilde{p})$ belongs to the set

$$
(\kappa, 0, \mu, 0,1-\mu, 1), \quad 0<\kappa, \mu<1, \kappa=n \mu .
$$

The latter set of exponents does not give us anything new as it essentially expresses a special case of the decay estimate

$$
\|U(t) f\|_{L_{t}^{q, \infty} L_{x}^{\infty} L_{v}^{n q}} \lesssim\|f\|_{L_{x}^{n q} L_{v}^{\infty}} .
$$

Let us use the more natural notation for the exponents $b=\tilde{r}^{\prime}$ and $c=\tilde{p}^{\prime}$. Thus we get the following system of conditions

$$
\begin{array}{cl}
\frac{1}{q}+\frac{n}{r}=\frac{n}{b}, & \operatorname{HM}(p, r)=\operatorname{HM}\left(\tilde{p}^{\prime}, \tilde{r}^{\prime}\right), \\
r<\frac{n c}{n-1}, & p<b \leq c<r, \\
1<q<\infty, & 1 \leq p, \tilde{p}, r, \tilde{r}<\infty .
\end{array}
$$

Corollary 8.2. The estimate

$$
\|U(t) f\|_{L_{t}^{q} L_{x}^{r} L_{v}^{p}} \lesssim\|f\|_{L_{x}^{b} L_{v}^{c}}, \quad b \neq c,
$$


holds for all $f \in L_{x}^{b} L_{v}^{c}$ whenever

$$
\begin{gathered}
\frac{1}{q}+\frac{n}{r}=\frac{n}{b}, \quad p<b<a<c<r, \quad r<\frac{n}{n-1} c, \quad q \geq c, \\
\operatorname{HM}(r, p)=\operatorname{HM}(b, c) \stackrel{\text { def }}{=} a, \quad 1<q, b, c, r<\infty, \quad 1 \leq p<\infty .
\end{gathered}
$$

The $L_{t}^{q}$-norm in (76) can be replaced by the $L_{t}^{q, c}$-norm. In such case the assumption $q \geq c$ can be removed.

Proof. Each estimate in the statement of this corollary can be proved by interpolating two estimates (75) with the real method. Indeed, let us perturb slightly the exponents $q, b$, and $c$, keeping $r$ and $p$ fixed, in such a way that they remain in the range of validity of the estimates (75). For example, the perturbed exponents can be taken as follows

$$
\begin{array}{lll}
1 / q_{1}=1 / q+n / \epsilon, & 1 / b_{1}=1 / b+1 / \epsilon, & 1 / c_{1}=1 / c-n / \epsilon, \\
1 / q_{2}=1 / q-n / \epsilon, & 1 / b_{2}=1 / b-1 / \epsilon, & 1 / c_{2}=1 / c+n / \epsilon .
\end{array}
$$

We then interpolate by the real method with $(\theta, q)=(1 / 2, c)$, and make use of Lemma 5.11.

Let us remark that the case of $b=c$, excluded in Corollary 8.2, is not new and is considered in Theorem 2.4 .

Lemma 8.3. Suppose that $(q, r, p)$ and $(\infty, \tilde{r}, \tilde{p})$ are two jointly KT-acceptable exponent triplets and $1<\tilde{p}^{\prime} \leq q<\infty$. Then the estimate

$$
\|W(t) F\|_{L_{t}^{q} L_{x}^{r} L_{v}^{p}} \lesssim\|F\|_{L_{t}^{1} L_{x}^{\tilde{r}^{\prime}} L_{v}^{\tilde{p}^{\prime}}}
$$

holds for all $F \in L_{t}^{1} L_{x}^{\tilde{r}^{\prime}} L_{v}^{\tilde{r}^{\prime}}$. Similarly, if $(\infty, r, p)$ and $(\tilde{q}, \tilde{r}, \tilde{p})$ are two jointly KTacceptable exponent triplets and $1<\tilde{q}^{\prime} \leq p<\infty$, then the estimate

$$
\|W(t) F\|_{L_{t}^{\infty} L_{x}^{r} L_{v}^{p}} \lesssim\|F\|_{L_{t}^{\tilde{q}^{\prime}} L_{x}^{\tilde{r}^{\prime}} L_{v}^{\tilde{p}^{\prime}}}
$$

holds for all $F \in L_{t}^{\tilde{q}^{\prime}} L_{x}^{\tilde{r}^{\prime}} L_{v}^{\tilde{p}^{\prime}}$.

Proof. The lemma follows directly from Lemma 8.1, Corollary 8.2, and the Equivalence Theorem 2.8. The range of validity of these estimates is identical to that of the generalized homogeneous estimates except for the usual change of notation.

Let us verify that the range of the exponents is the same as that assumed in Theorem 2.5. The assumption there is that $(q, r, p)$ and $(\infty, \tilde{r}, \tilde{p})$ are jointly KTacceptable and that $1<q<\infty$. This immediately implies the following range

$$
1<q<\infty, \quad 1 \leq p, r, \tilde{p}, \tilde{r}<\infty .
$$

Next, the requirement that $(q, r, p)$ is KT-acceptable and that $q<\infty$ leads to $p<r$. Therefore $r>1$. The scaling condition (173) together with the fact that $(q, r, p)$ is KT-acceptable implies that $p<\tilde{r}^{\prime}$. The identity $\operatorname{HM}(p, r)=\operatorname{HM}(\tilde{p}, \tilde{r})$ together with $p<r$, and $\tilde{p} \leq \tilde{r}$, and $p<\tilde{r}^{\prime}$, leads to

$$
p<\tilde{r}^{\prime} \leq \tilde{p}^{\prime}<r .
$$

The latter implies that $1<\tilde{p} \leq \tilde{r}<\infty$. Thus we obtain the following range

$$
1<q, r, \tilde{p}, \tilde{r}<\infty, \quad 1 \leq p<\infty,
$$

which is the range for which the estimates in this lemma are proven. Analogously for the dual case. 
8.3. Global inhomogeneous endpoint estimates with $q=\tilde{q}^{\prime}$. In this paragraph we assume that the $L_{v}^{p}$-norms are given over a bounded velocity space $V \subset \mathbb{R}^{n}$ and prove the inhomogeneous estimates (20).

We suppose now that $P$ lies on the hypotenuse of $\triangle O A B$ in Figure 1 and that it also belongs to $\mathcal{E}_{0}$. The 4 -vector $(1 / r, 1 / p, 1 / \tilde{r}, 1 / \tilde{p})$ should satisfy every inequality in $\mathcal{E}_{0}$ as a strict inequality. Of course, we cannot remove the restriction $\operatorname{HM}(p, r)=$ $\operatorname{HM}\left(\tilde{p}^{\prime}, \tilde{r}^{\prime}\right)$, but we shall perturb these exponents in such a way that they always satisfy the latter condition. The exponents $(1 / q, 1 / \tilde{q})$ will remain fixed throughout this paragraph. We consider the following perturbations

$$
\begin{array}{llll}
\frac{1}{r_{0}}=\frac{1}{r}+\epsilon, & \frac{1}{\tilde{r}_{0}}=\frac{1}{\tilde{r}}+\epsilon, & \frac{1}{p_{0}}=\frac{1}{p}-\epsilon, & \frac{1}{\tilde{p}_{0}}=\frac{1}{\tilde{p}}-\epsilon, \\
\frac{1}{r_{1}}=\frac{1}{r}-3 \epsilon, & \frac{1}{\tilde{r}_{1}}=\frac{1}{\tilde{r}}-3 \epsilon, & \frac{1}{p_{1}}=\frac{1}{p}+3 \epsilon, & \frac{1}{\tilde{p}_{1}}=\frac{1}{\tilde{p}}+3 \epsilon .
\end{array}
$$

We have that $\beta\left(q, r_{0}, \tilde{q}, \tilde{r}_{0}\right)=2 n \epsilon$ and $\beta\left(q, r_{1}, \tilde{q}, \tilde{r}_{0}\right)=\beta\left(q, r_{0}, \tilde{q}, \tilde{r}_{1}\right)=-2 n \epsilon$. Hence the maps

$$
\begin{aligned}
& A: L_{t}^{\tilde{q}^{\prime}} L_{x}^{\tilde{r}_{0}^{\prime}} L_{v}^{\tilde{p}_{0}^{\prime}} \times L_{t}^{q^{\prime}} L_{x}^{r_{0}^{\prime}} L_{v}^{p_{0}^{\prime}} \rightarrow l_{-2 \epsilon}^{\infty}, \\
& A: L_{t}^{\tilde{q}^{\prime}} L_{x}^{\tilde{r}_{0}^{\prime}} L_{v}^{\tilde{p}_{0}^{\prime}} \times L_{t}^{q^{\prime}} L_{x}^{r_{1}^{\prime}} L_{v}^{p_{1}^{\prime}} \rightarrow l_{2 \epsilon}^{\infty}, \\
& A: L_{t}^{\tilde{q}^{\prime}} L_{x}^{\tilde{r}_{1}^{\prime}} L_{v}^{\tilde{p}_{1}^{\prime}} \times L_{t}^{q^{\prime}} L_{x}^{r_{0}^{\prime}} L_{v}^{p_{0}^{\prime}} \rightarrow l_{2 \epsilon}^{\infty},
\end{aligned}
$$

are bounded. In virtue of Lemma 5.14 and the well-known interpolation identity

$$
\left(L^{p}\left(\mathbb{R} ; \mathcal{A}_{0}\right), L^{p}\left(\mathbb{R} ; \mathcal{A}_{1}\right)\right)_{\theta, p}=L^{p}\left(\mathbb{R} ;\left(\mathcal{A}_{0}, \mathcal{A}_{1}\right)_{\theta, p}\right), \quad 1<p<\infty,
$$

see [3], the map

$$
\begin{aligned}
A:\left(L_{t}^{\tilde{q}^{\prime}} L_{x}^{\tilde{r}_{0}^{\prime}} L_{v}^{\tilde{p}_{0}^{\prime}}, L_{t}^{\tilde{q}^{\prime}} L_{x}^{\tilde{r}_{1}^{\prime}} L_{v}^{\tilde{p}_{1}^{\prime}}\right)_{1 / 4, \tilde{q}^{\prime} \times} & \left(L_{t}^{q^{\prime}} L_{x}^{r_{0}^{\prime}} L_{v}^{p_{0}^{\prime}}, L_{t}^{q^{\prime}} L_{x}^{r_{1}^{\prime}} L_{v}^{p_{1}^{\prime}}\right)_{1 / 4, q^{\prime}} \rightarrow\left(l_{2 \epsilon}^{\infty}, l_{-2 \epsilon}^{\infty}\right)_{1 / 2,1}
\end{aligned}
$$

is also bounded. In view of the fact that $V$ is bounded we have that $L^{\tilde{P}^{\prime}}(V) \hookrightarrow$ $L^{\tilde{p}_{0}^{\prime}}(V)$ and $L^{\tilde{P}}(V) \hookrightarrow L^{\tilde{p}_{1}^{\prime}}(V)$ whenever $1 \leq \tilde{P} \leq \min \left(\tilde{p}_{0}, \tilde{p}_{1}\right)$. Analogously, $L^{P^{\prime}}(V) \hookrightarrow L^{p_{0}^{\prime}}(V)$ and $L^{P^{\prime}}(V) \hookrightarrow L^{p_{1}^{\prime}}(V)$ whenever $1 \leq P \leq \min \left(p_{0}, p_{1}\right)$. Thus we also have that the map

$$
\begin{aligned}
A:\left(L_{t}^{\tilde{q}^{\prime}} L_{x}^{\tilde{r}_{0}^{\prime}} L_{v}^{\tilde{P}^{\prime}}, L_{t}^{\tilde{q}^{\prime}} L_{x}^{\tilde{r}_{1}^{\prime}} L_{v}^{\tilde{P}^{\prime}}\right)_{1 / 4, \tilde{q}^{\prime} \times} & \left(L_{t}^{q^{\prime}} L_{x}^{r_{0}^{\prime}} L_{v}^{P^{\prime}}, L_{t}^{q^{\prime}} L_{x}^{r_{1}^{\prime}} L_{v}^{P^{\prime}}\right)_{1 / 4, q^{\prime}} \rightarrow\left(l_{2 \epsilon}^{\infty}, l_{-2 \epsilon}^{\infty}\right)_{1 / 2,1}
\end{aligned}
$$

is bounded. Finally, in view of the interpolation identity (77), it follows that

$$
A: L_{t}^{\tilde{q}^{\prime}} L_{x}^{\tilde{r}^{\prime}, \tilde{q}^{\prime}} L_{v}^{\tilde{P}^{\prime}} \times L_{t}^{q^{\prime}} L_{x}^{r^{\prime}, q^{\prime}} L_{v}^{P^{\prime}} \rightarrow l^{1} .
$$

In view of Lemma 5.9, this implies the estimate

$$
\|W(t) F\|_{L_{t}^{q} L_{x}^{r, q} L_{v}^{P}(V)} \lesssim_{V}\|F\|_{L_{t}^{\tilde{q}^{\prime}} L_{x}^{\tilde{r}^{\prime}, \tilde{q}^{\prime}} L_{v}^{\tilde{P}^{\prime}}(V)},
$$

for any $P, \tilde{P}$, such that $1 \leq P<p$ and $1 \leq \tilde{P}<\tilde{p}$, and any two jointly KTacceptable exponent triplets $(q, r, p)$ and $(\tilde{q}, \tilde{r}, \tilde{p})$ whose exponents further satisfy the following conditions $1<q, \tilde{q}<\infty, q=\tilde{q}^{\prime}$. 


\section{Counterexamples}

In this section we give necessary conditions for the range of validity of the Strichartz estimates for the KT equation by means of counterexamples.

We first make the general remark that the validity of Strichartz estimates with exponents $r=\infty$ in the homogeneous setting, and with $r=\infty$ or $\tilde{r}=\infty$ in the inhomogeneous setting, is completely solved in [12. There it is proved that the only valid estimate of the form

$$
\|U(t) f\|_{L_{t}^{q} L_{x}^{\infty} L_{v}^{p}} \lesssim\|f\|_{L_{x}^{b} L_{v}^{c}}, \quad \forall f \in L_{x}^{b} L_{v}^{c}
$$

in any spatial dimension is for $q=p=b=c=\infty$. Also, the only valid inhomogeneous estimates of the form

$$
\|W(t) F\|_{L_{t}^{q} L_{x}^{r} L_{v}^{p}} \lesssim\|F\|_{L_{t}^{\tilde{q}^{\prime}} L_{x}^{\tilde{r}^{\prime}} L_{v}^{\tilde{p}^{\prime}}}, \quad \forall F \in L_{t}^{\tilde{q}^{\prime}} L_{x}^{\tilde{r}^{\prime}} L_{v}^{\tilde{p}^{\prime}},
$$

in any spatial dimension with either $r=\infty$ or $\tilde{r}=\infty$ are only those whose exponents are explicitly stated in Definition 2.3 .

9.1. Homogeneous estimates. By scaling, that is Lemma 4.5, estimate

$$
\|U(t) f\|_{L_{t}^{q} L_{x}^{r} L_{v}^{p}} \lesssim\|f\|_{L_{x, v}^{a}}, \quad \forall f \in L_{x, v}^{a},
$$

holds only if

$$
\frac{1}{q}+\frac{n}{r}=\frac{n}{a}, \quad a=\operatorname{HM}(p, r)
$$

Let us next find the upper bound $r \leq r^{*}(a)$. It is enough to consider only the special case $a=2$. We shall prove the equivalent condition $q \geq 2$. (In general $r \leq r^{*}(a)$ and $q \geq a$ are equivalent.) The claim follows directly by the translation invariance in $t$ of the $T T^{*}$-operator. Indeed, first recall that the above estimate with $a=2$ is equivalent to

$$
\left\|T T^{*} F\right\|_{L_{t}^{q} L_{x}^{r} L_{v}^{r^{\prime}}} \lesssim\|F\|_{L_{t}^{q^{\prime}} L_{x}^{r^{\prime}} L_{v}^{r}}, \quad \forall F \in L_{t}^{q^{\prime}} L_{x}^{r} L_{v}^{r^{\prime}}
$$

Then, in view of the famous Hörmander's lemma 9.1, we have that $q \geq q^{\prime}$, or equivalently $q \geq 2$.

For finding a lower bound on $r$ we use the translation invariance in $x$ of $T T^{*}$ and thus we get that $r \geq r^{\prime}$, or equivalently, $r \geq 2$. As usual, the condition $r \geq a$ in the general case $0<a<\infty$ follows by the power invariance (34).

Let us verify the translation invariance in $t$ of $T T^{*}$. Consider $F_{\tau}(t)=F(t-\tau)$. For $T T^{*} F_{\tau}$ we have

$$
\int_{-\infty}^{\infty} U(t-s) F(s-\tau) d s=\int_{-\infty}^{\infty} U(t-\tau-\sigma) F(\sigma) d \sigma
$$

or in other words $\left\{T T^{*} F_{\tau}\right\}(t)=\left\{T T^{*} F\right\}(t-\tau)$.

Lemma 9.1 (Hörmander [9]). Whenever a (non-trivial) linear and bounded operator maps $L^{p}\left(\mathbb{R}^{n}\right)$ to $L^{q}\left(\mathbb{R}^{n}\right), 1 \leq p, q<\infty$, and additionally this operator is translation invariant, then we must have that $p \leq q$.

Remark 9.2. Hörmander's lemma remains true in a more general setting. For example, the space $L^{p}$ and $L^{q}$ can be vector-valued, i.e. $L^{p}\left(X ; \mathcal{B}_{1}\right)$ and $L^{q}\left(X ; \mathcal{B}_{1}\right)$ respectively, where $X \subseteq \mathbb{R}^{n}$ is the set $\left\{x=\left(x_{1}, \ldots, x_{n}\right) \mid a_{i}<x_{i}<\infty, i=1, \ldots n\right\}$ for some fixed $a_{i} \in \mathbb{R} \cup\{-\infty\}$, and $\mathcal{B}_{1}$ and $\mathcal{B}_{2}$ are some Banach spaces. Furthermore, the 
spaces $L^{p}$ and $L^{q}$ may be mixed Lebesgue spaces (or Bochner spaces in the vectorvalued setting). Suppose for example that $p=\left(p_{1}, \ldots, p_{k}\right)$ and $q=\left(q_{1}, \ldots, q_{l}\right)$ and $L^{p}$ and $L^{q}$ are the corresponding mixed Lebesgue spaces with the usual notation. Consider the bounded linear operator $T: L^{p} \rightarrow L^{q}$. Let $u\left(x_{1}, \ldots, x_{k}\right) \in L^{p}$, $v\left(y_{1}, \ldots, y_{l}\right) \in L^{q}, \tau_{h}$ be the operator defined by

$$
\tau_{h} u\left(x_{1}, \ldots, x_{i}, \ldots, x_{k}\right)=u\left(x_{1}, \cdots, x_{i}+h, \ldots, x_{k}\right),
$$

and similarly let $\sigma_{h}$ be the operator defined by

$$
\sigma_{h} v\left(y_{1}, \ldots, y_{j}, \ldots, y_{l}\right)=v\left(y_{1}, \ldots, y_{j}+h, \ldots, y_{l}\right) .
$$

Then, if we have that

$$
T \tau_{h} u=\sigma_{h} T u, \quad \forall h \geq 0,
$$

it follows that either $q_{j} \geq p_{i}$, or $T=0$. The proof of that statement is virtually the same as that of Lemma 9.1 .

9.2. Generalized homogeneous estimates. Let us consider the homogeneous Strichartz estimate

$$
\|U(t) f\|_{L_{t}^{q} L_{x}^{r} L_{v}^{p}} \lesssim\|f\|_{L_{x}^{b} L_{v}^{c}}, \quad \forall f \in L_{x}^{b} L_{v}^{c}
$$

for data outside the transport class. Most of the arguments from the preceding paragraph apply to this case as well. By scaling, we have that the conditions

$$
\frac{1}{q}+\frac{n}{r}=\frac{n}{b}, \quad \operatorname{HM}(p, r)=\operatorname{HM}(b, c) \stackrel{\text { def }}{=} a,
$$

are necessary. The following conditions

$$
r \geq p, \quad \frac{1}{q}<n\left(\frac{1}{p}-\frac{1}{r}\right), \quad \text { or } q=\infty, 1 \leq p=r \leq \infty,
$$

are also necessary. To that end, let us consider the equivalent estimate

$$
\left\|T T^{*} F\right\|_{L_{t}^{q} L_{x}^{r} L_{v}^{p}} \lesssim\|F\|_{L_{t}^{1} L_{x}^{b} L_{v}^{c}}, \quad \forall F \in L_{t}^{1} L_{x}^{b} L_{v}^{c} .
$$

The claim is proved for it in the next paragraph. Analogously, we obtain that $b \leq c$. Indeed, the latter estimate is equivalent to

$$
\left\|T T^{*} F\right\|_{L_{t}^{\infty} L_{x}^{b^{\prime}} L_{v}^{c^{\prime}}} \lesssim\|F\|_{L_{t}^{q^{\prime}} L_{x}^{r^{\prime}} L_{v}^{p^{\prime}}}, \quad \forall F \in L_{t}^{q^{\prime}} L_{x}^{r^{\prime}} L_{v}^{p^{\prime}} .
$$

The exponent triplet must be KT-acceptable (proved in the next paragraph) and thus $b^{\prime} \geq c^{\prime}$. In fact, conditions (80) and (79) imply that either $p<b \leq a \leq c<r$ $(p<b)$, or $a=b=c=p=r$ and $q=\infty$.

We do not have a suitable counterexample showing the necessity of the upper bound $r^{*}(c)$ in Theorem 2.10 for the validity of the generalized homogeneous estimates (in the case when $b \neq c, n>1$ ).

9.3. Generalized inhomogeneous estimates. Let us consider now the inhomogeneous Strichartz estimate

$$
\|W(t) F\|_{L_{t}^{q} L_{x}^{r} L_{v}^{p}} \lesssim\|F\|_{L_{t}^{\tilde{q}^{\prime}} L_{x}^{\tilde{r}^{\prime}} L_{v}^{\tilde{p}^{\prime}}}
$$

By scaling, see Lemma 4.5, we obtain that the restrictions

$$
\frac{1}{q}+\frac{1}{\tilde{q}}=n\left(1-\frac{1}{r}-\frac{1}{\tilde{r}}\right), \quad \operatorname{HM}(p, r)=\operatorname{HM}\left(\tilde{p}^{\prime}, \tilde{r}^{\prime}\right) \stackrel{\text { def }}{=} a,
$$

are necessary. 
Consider $F(t, x, v)=\chi(0 \leq t \leq 1,|x| \leq 1,|v| \leq 1)$. When $t \gg 1$ we have that

$$
\left\{T T^{*} F\right\}(t)=W(t) F \approx \chi\left(\left|v-\frac{x}{t}\right| \leq \frac{1}{t},|v| \leq 1\right) \approx \chi\left\{v \sim \frac{1}{t}, x \sim t\right\} .
$$

Hence,

$$
\|W(t) F\|_{L_{x}^{r} L_{v}^{p}} \sim t^{\frac{n}{r}-\frac{n}{p}}, \quad t \gg 1 .
$$

It follows that $\|W(t) F\|_{L_{t}^{q} L_{x}^{r} L_{v}^{p}}<\infty$ only if

$$
\left(\frac{n}{r}-\frac{n}{p}\right) q<-1, \quad \text { or if } q=\infty, r=p .
$$

By the duality Lemma 5.3 , the dual exponents $(\tilde{q}, \tilde{r}, \tilde{p})$ must also satisfy (81). Thus we have that the conditions $p \leq r$ and $\tilde{p} \leq \tilde{r}$ are necessary for the validity of the considered estimate. The same conclusion applies for the $T T^{*}$-operator.

We now show that conditions

$$
\frac{1}{q}+\frac{1}{\tilde{q}} \leq 1, \quad \frac{1}{r}+\frac{1}{\tilde{r}} \leq 1,
$$

are necessary for the validity of the considered estimate. Indeed, the claim follows from the translation invariance of $T T^{*}$ in $t$ and $x$, Hörmander's lemma 9.1, and the equivalence of the considered estimates for $T T^{*}$ and $W(t)$. Note also that the cases when $\tilde{q}=1$ or $\tilde{r}=1$ are trivial and for example by duality can always be replaced by the cases $q=1$ or $r=1$. Thus we have verified that $(q, r, p)$ and $(\tilde{q}, \tilde{r}, \tilde{p})$ must be two jointly KT-acceptable exponent triplets, apart from the necessity of condition

$$
\frac{n-1}{p^{\prime}}<\frac{n}{\tilde{r}}, \quad \frac{n-1}{\tilde{p}^{\prime}}<\frac{n}{r}, \quad n>1
$$

for which we do not have a suitable counterexample. However, we can show that the similar condition

$$
\frac{n}{p^{\prime}}<\frac{1}{\tilde{q}}+\frac{n}{\tilde{r}}, \quad \frac{n}{\tilde{p}^{\prime}}<\frac{1}{q}+\frac{n}{r},
$$

is sharp. Indeed, the latter is a direct consequence of (10) and (11). The latter condition implies the former whenever $p^{\prime} \leq \tilde{q}$ and $\tilde{p}^{\prime} \leq q$. Thus, if there are some other global inhomogeneous estimates for $W(t)$ not included in Theorem 2.5 they must belong to the range $\tilde{q}<p^{\prime}$ or $q<\tilde{p}^{\prime}$.

9.4. Local inhomogeneous estimates. In this paragraph we show the fact that in the context of the KT equation the local inhomogeneous estimates do not exist in a "full neighborhood" around a given local inhomogeneous Strichartz estimate. This presents an obstruction for the application of the perturbation techniques of Keel and Tao 11 and their extension by Foschi 7 . The endpoint estimates (of the type that lie on the hypothenuse $A B$ in Figure 1) remain unresolved.

For example, consider the estimate

$$
\|W(t) F\|_{L_{t}^{q}\left([2,3] ; L_{x}^{r} L_{v}^{p}\right)} \lesssim\|F\|_{L_{t}^{\tilde{q}^{\prime}}\left([1,2] ; L_{x}^{\tilde{r}^{\prime}} L_{v}^{\tilde{p}^{\prime}}\right)} .
$$

Take $F(t, x, v)=\chi\left(t \in[0,1],(x, v) \in Q_{R}\right)$, where by $Q_{R}$ we denote the square of side length $2 R$ centered at the origin of $\mathbb{R}^{2 n}$. If we denote $\|x\|_{\infty}=\sup _{1 \leq i \leq n}\left|x_{i}\right|$, for $x=\left(x_{1}, . ., x_{n}\right)$, we can write the latter as

$$
Q_{R}=\left\{(x, v):\|x\|_{\infty} \leq R,\|v\|_{\infty} \leq R\right\} .
$$

Hence,

$$
\|F\|_{L_{t}^{\tilde{q}^{\prime}}\left([1,2] ; L_{x}^{\tilde{r}^{\prime}} L_{v}^{\tilde{p}^{\prime}}\right)} \sim R^{\frac{n}{\tilde{p}^{\prime}}+\frac{n}{\tilde{r}^{\prime}}} .
$$


We now set $\tau=t-s$, and consider the set $Q_{R}(\tau)$ given by

$$
\|x-\tau v\|_{\infty} \leq R,\|v\|_{\infty} \leq R .
$$

Then, for $t \in[2,3], s \in[0,1]$, equivalently for $\tau \in[1,3]$, we have the inclusions

$$
Q_{R / 4} \subset Q_{R}(\tau) \subset Q_{4 R} .
$$

Hence,

We conclude that condition

$$
\|W(t) F\|_{L_{t}^{q}\left([2,3] ; L_{x}^{r} L_{v}^{p}\right)} \sim R^{\frac{n}{p}+\frac{n}{r}} .
$$

$$
\frac{1}{r}+\frac{1}{p}=\frac{1}{\tilde{r}^{\prime}}+\frac{1}{\tilde{p}^{\prime}}
$$

is necessary for the validity of the local estimates (82).

\section{Remaining unResolved Strichartz estimates}

Here we collect some of the remaining estimates for the KT equation that need to be resolved in order the full range of validity of Strichartz estimates to be known.

(1) The endpoint homogeneous estimate in higher dimensions $n>1$

$$
\|U(t) f\|_{L_{t}^{a} L_{x}^{r^{*}(a)} L_{v}^{p^{*}(a)}} \lesssim\|f\|_{L_{x, v}^{a}} .
$$

(2) The full range of the non-endpoint inhomogeneous estimates

$$
\|W(t) F\|_{L_{t}^{q} L_{x}^{r} L_{v}^{p}} \lesssim\|F\|_{L_{t}^{\tilde{q}^{\prime}} L_{x}^{\tilde{r}^{\prime}} L_{v}^{\tilde{p}^{\prime}}}, \quad q>\tilde{q}^{\prime} .
$$

In particular, one needs to either show that the condition

$$
\frac{n-1}{p^{\prime}}<\frac{n}{\tilde{r}}, \quad \frac{n-1}{\tilde{p}^{\prime}}<\frac{n}{r},
$$

is necessary, or otherwise find and prove the remaining estimates.

(3) The endpoint inhomogeneous estimates with either $q=\tilde{q}^{\prime}$ or $q=\infty$ or $\tilde{q}=\infty$.

(4) The full range of the local inhomogeneous estimates. Equivalently, either show that $\mathcal{E}_{0}=\mathcal{E}$, or otherwise find and prove the remaining estimates.

\section{REFERENCES}

1. A. Benedek and R. Panzone, The space $L^{p}$, with mixed norm., Duke Math. J. 28 (1961), no. $2,301-324$.

2. Colin Bennett and Robert Sharpley, Interpolation of operators, Pure and Applied Mathematics, 129, Academic Press, Inc., Boston, MA, 1988.

3. Jöran Bergh and Jörgen Löfström, Interpolation spaces. an introduction., Grundlehren der Mathematischen Wissenschaften, No. 223. Springer-Verlag, Berlin-New York, 1976.

4. Nikolaos Bournaveas, Vincent Calvez, Susana Gutiérrez, and Benoît Perthame, Global existence for a kinetic model of chemotaxis via dispersion and Strichartz estimates, Comm. Partial Differential Equations 33 (2008), no. 1-3, 79-95.

5. F. Castella and B. Perthame, Estimations de Strichartz pour les équations de transport cinétique, C. R. Acad. Sci. Paris, Ser. I (1996), no. 332, 535-540.

6. Elena Cordero and Fabio Nicola, Some new Strichartz estimates for the Schrödinger equation., Preprint (2007), available at http://arxiv.org.

7. Damiano Foschi, Inhomogeneous Strichartz estimates, J. Hyperbolic Differ. Equ. 2 (2005), no. 1, 1-24.

8. Zihua Guo and Lizhong Peng, Endpoint Strichartz estimate for the kinetic transport equation in one dimension, C. R. Math. Acad. Sci. Paris 345 (2007), no. 5, 253-256.

9. L. Hörmander, Estimates for translation invariant operators in $L^{p}$ spaces., Acta Math. 104 (1960), no. 5, 93-140. 
10. Tosio Kato, An $L^{q, r}$-theory for nonlinear Schrödinger equations., Spectral and Scattering Theory and Applications, Adv. Stud. Pure Math. 23 (1994), 223-238.

11. Markus Keel and Terence Tao, Endpoint Strichartz estimates, Amer. J. Math. 120 (1998), no. 5, 955-980.

12. Evgeni Y Ovcharov, Counterexamples to Strichartz Estimates for the Kinetic Transport Equation based on Besicovitch sets, Preprint (2010).

13. — Global regularity of dispersive equations and Strichartz estimates., Ph.D. Thesis (2009), the University of Edinburgh.

14. Benoît Perthame, Mathematical tools for kinetic equations., Bull. Amer. Math. Soc. (N.S.) 41 (2004), no. 2, 205-244.

15. Sigmund Selberg, Lecture notes on nonlinear wave equations, Personal web site: http://www.math.ntnu.no/ sselberg/ (2001).

16. Robert J Taggart, Inhomogeneous Strichartz estimates., Preprint (2008), http://arxiv.org.

17. M.C. Vilela, Inhomogeneous Strichartz estimates for the Schrödinger equation, Trans. Amer. Math. Soc. 359 (2007), no. 5, 2123-2136.

Angewandte Mathematik und Bioquant, Universität Heidelberg, INF 267, Heidelberg 69120, Germany

E-mail address: evgeni.ovcharov@bioquant.uni-heidelberg.de 